\title{
Dynamic changes in semantic memory structure support successful problem-solving
}

\author{
Affiliation: \\ ${ }^{1}$ Sorbonne University, Institut du Cerveau - Paris Brain Institute -ICM-, Inserm, CNRS, AP- \\ HP Hôpital de la Pitié-Salpêtrière, Paris, France \\ ${ }^{2}$ Neurology department, Pitié-Salpêtrière hospital, AP-HP, F-75013, Paris, France \\ ${ }^{3}$ Faculty of Industrial Engineering and Management, Technion - Israel Institute of \\ Technology, Haifa 3200003 Israel \\ ${ }^{4}$ Sleep center, Pitié-Salpêtrière hospital, AP-HP, F-75013, Paris, France \\ * equal contribution

\section{Corresponding authors:} \\ Emmanuelle Volle (emmavolle@gmail.com) \\ Théophile Bieth (theo bieth@hotmail.fr)
}

\section{Key words:}

Problem-solving, Semantic networks, Restructuration, Cognitive mechanism, Creativity

Title: 9 words

Abstract: 158 words

Main manuscript (without references): 7385 words

Method section: 2564 words

Figure: 4

Table: 0

Supplementary material and results: 1138 words

Supplementary figures: 5

Supplementary table: 4 
While problem-solving is central in our daily life, its underlying mechanisms remain largely unknown. Restructuration (i.e., reinterpretation and reorganization of problem-related representations) is theoretically considered as one such mechanism, yet empirical evidence supporting it is scarce. We investigated restructuration as a mechanism underlying problemsolving, using network science methodology. We estimated the structure of participant's individual semantic memory network before and after they attempted to solve a riddle. These networks represent the organization of solution-relevant and irrelevant terms as nodes, with edges representing the strength of relationship between them based on participants' relatedness judgments. The difference in semantic network metrics between pre- and post-solving phases was used to quantify restructuration and predict successful problem-solving. Problem-solving was predicted by local restructuration of semantic network, only in edges and nodes that had been assessed as helpful to solve the problem. These results shed new light on the mental restructuring associated with problem-solving and provide a new method to quantify this restructuring. 


\section{Introduction}

In our daily life, we constantly deal with new problems, ranging from the most mundane (e.g., what to cook for dinner given the ingredients at our disposal), to professional activities (e.g., how to reorganize our current plans to meet a new deadline), to the biggest societal challenges (e.g., how to find innovative solutions against global warming). While the ability to solve problems is a critical skill adapting to new situations and innovating, the mechanisms underlying the problem-solving process remain largely unknown. How do we find new and adapted solutions to problems that we never encountered before?

Among the problems we face each day, some are well-defined (e.g., playing chess) in the sense that the initial state (i.e., the chess board) and goal state (i.e., checkmate) are clear, and the solver can apply a set of operations (i.e., chest moves) to reach the goal. However, for most of our problems (e.g., organizing work activities during the COVID-19 pandemic), the problem space is ambiguous. There are no heuristics or existing rules that could easily be applied to transform the initial state into the goal state ${ }^{1}$. Such ill-defined problems ${ }^{2}$ thus require additional mental processes, which have been tightly linked to creative thinking ${ }^{3-5}$. Ill-defined problem-solving (or creative problem-solving) often referred to insight-solving, where the solution came to mind suddenly, spontaneously, and effortlessly, with a "Eureka" phenomenon ${ }^{6-8}$. They are thought to involve a restructuring of the initial problem mental representational space $^{3}$, that presumably entails combining elements related to the problem in a new way or creating novel and unexpected connections between remote concepts stored in semantic memory ${ }^{9,10}$. It ultimately allows the solver to change perspective, reframe the problem, or escape its implicitly imposed constraints ${ }^{11}$ and leads to creative associations ${ }^{7,9}$. For instance, consider the following problem: "A man walks into a bar and asks for a glass of water. The bartender points a shotgun at the man. The man says, 'Thank you,' and walks out" ${ }^{12}$. How to make sense of this story that seems to be missing a piece? The problem is ill-defined because the path to find the solution is left for participants to identify, and the goal state is vague. Solving this problem first requires asking the right question: in which context would a shotgun and a glass of water help somebody? Rather than relying on obvious associations (e.g., a glass of water is related to thirst), solvers must fill the missing link between the relevant elements of the problem (a shotgun induces fear plus fear and drinking water are two remedies for hiccups). Restructuring one's existing semantic associations would allow to see this link and find the solution.

Although this hypothesis is intuitively satisfying, empirical evidence supporting it is surprisingly scarce ${ }^{12-14}$. The assumption that solvers changed or restructured their internal representation of the problem often stems from the mere fact that they found the solutions (circular argument), rather than from a direct measurement of the restructuring process ${ }^{15}$. Here, we propose to quantify problem restructuring, using tools from network science.

Network science is based on mathematical graph theory, offering quantitative tools and methods to represent complex systems as graphs or networks ${ }^{16}$. A growing amount of research has been applying network science methods to study cognitive systems, mainly focusing on memory and language ${ }^{16,17}$. Specifically, SemNets represent elements of knowledge as nodes related to each other with edges of various strengths ${ }^{18-20}$. They have been used to explore the properties and organization of semantic memory $16,20-22$, and how this organization relates to creativity ${ }^{23-30}$. Importantly, Schilling ${ }^{10}$ has theoretically argued that insight solving occurs as a result of restructuring of the semantic memory network structure, through the creation of new links between nodes in semantic memory. We propose that SemNets can be used to model how the elements of a new problem are semantically associated with one another in a person's mind and how changes in these associations predict successful problem-solving ${ }^{31,32}$. 
Using the riddle presented above, Durso et al. ${ }^{12}$ empirically demonstrated a restructuration of the solution-relevant semantic associations in the participants who solved the problem. This was achieved by having participants who solved (solvers) or did not solve (nonsolvers) the riddle make relatedness judgments for all possible pairs of a set of 14 words related or not to this riddle. The pathfinder algorithm was used to compute group-based SemNets. The authors showed that semantic associations between specific problem elements differ between the solver and non-solver groups. In addition, the authors showed how edges between nodes that are decisive to solving the puzzle are gradually identified as critical, and that such problem reorganization was associated with solving of the puzzle ${ }^{12}$. Thus, the authors claimed their findings provide clear evidence for active restructuring during creative problem-solving and that such restructuring happens prior to successfully solving the creative problem ${ }^{12}$. Currently, no replication of the Durso et al. study ${ }^{12}$ has been conducted. Additionally, Durso et al. ${ }^{12}$ did not explored SemNet structure at the individual level. Thus, applying a current SemNet approach, recently applied in creativity research, would allow overcoming these limitations and better capture the restructuring of associations and problem representations related to successful creative problem-solving $25,26,29,30$.

Our study aims to explore the restructuring of SemNets as a cognitive mechanism underlying successful problem-solving and characterize this restructuring using network science methodologies. For this purpose, we applied a novel SemNet method to estimate individual SemNets across two different time points: once before the presentation of a problem, operationalized as a riddle (such as the one described above), and again after an attempt to solve it. We hypothesized that individual changes in SemNet organization (i.e., restructuring) would predict success in solving the problem. In particular, we hypothesized that edges or nodes that are the most relevant to the solution would become more connected or central in individual SemNets.

\section{Results}

Creative problem-solving ability was operationalized in an experimental procedure using four riddles labeled "Zoe", "Daniel", "Car" and "Bar" riddles (Table S1 and S2; see methods). For example, the Zoe riddle states the following: "Zoe throws a stone that lands in the sky. How is it possible and in which context?" and the Bar riddle is the one used by Durso et al. ${ }^{12}$. Participants $(n=99)$ were asked to solve two riddles one at a time (Figure 1). The order of the riddles was counterbalanced between participants and per couple of riddles (four possible combinations: Zoe/Daniel, $\mathrm{n}=25$, Daniel/Zoe, $\mathrm{n}=24$; Bar/Car, $\mathrm{n}=25$; and Car/Bar, $\mathrm{n}=25$ ). For each riddle, participants had a 10-minute active thinking phase during which they were encouraged to give as many guesses as possible without self-censoring (i.e., to indicate even inadequate or bizarre ideas that came to mind), rate their confidence in each response, and indicate whether they experienced a Eureka feeling. Furthermore, we regularly generated probes to assess participants' subjective level of focus on the task (Figure 1).

For each riddle, we estimated individual SemNet before the presentation of the riddle (Pre-SemNet) and after the solving attempt (Post-SemNet), using a Related Judgment task (RJT) ${ }^{25}$ (Figure 1). In this task, participants were asked to rate the semantic relatedness of word pairs. We built a riddle-specific material for the RJT. Hence, each riddle was assigned a unique set of 20 words, and the relatedness of all possible pairs of these 20 words was rated during the RJT (190 ratings; see methods). For each riddle-specific RJT, we selected words related to the riddle or its solution and unrelated words (see supplementary material). For example, in the Zoe riddle, solution-related words included to jump or drawing while unrelated words included gravity or plane (see Table S3). 
Based on previous studies that have related individual SemNets to creativity ${ }^{29,30}$, the RJT ratings were used to estimate individual weighted undirected SemNets (WUN), in which the nodes were the preselected riddle-related words and the edges represented the ratings during the RJT. In WUN, all links between the words (nodes) were weighted using the individual RJT ratings. For pre-and post-solving SemNet, we computed established network-based metrics that quantify the structural properties of each SemNet at a global level (i.e., describing the entire network structure) ${ }^{16,23,24,33}$ and at the local level (i.e., describing the properties of nodes or edges). Global metrics included: efficiency (Eff global; measuring how efficiently the SemNet exchanges information), clustering coefficient (CC global; measuring overall connectivity in the SemNet), and modularity (Q $\mathrm{Q}_{\text {global }}$; measuring the level of segregation of the SemNet). Local metrics were computed at the node level to estimate the connectivity and centrality of each node in the network: $\mathrm{CC}_{\text {local }}$ and eigenvector centrality $\left(\mathrm{EV}_{\text {local }}\right.$; a self-referential measure of centrality); as well as at the edge level to estimate the importance and efficiency of each edge in the network (weight $-\mathrm{W}_{\text {local, }}$, and $\mathrm{Eff}_{\text {local }}$ ). For each participant, we focused on SemNet metric changes between Pre-SemNet (i.e., before the problem) and Post-SemNet (i.e., after the solving attempt). We also took into account the importance of the different edges and nodes to solve the riddle, as determined by independent assessments of external judges (see methods). These 'impact ratings' allowed us to weight edges and nodes according to their relevance for solving the problem: the higher the impact rating, the more helpful the edges or nodes we considered.

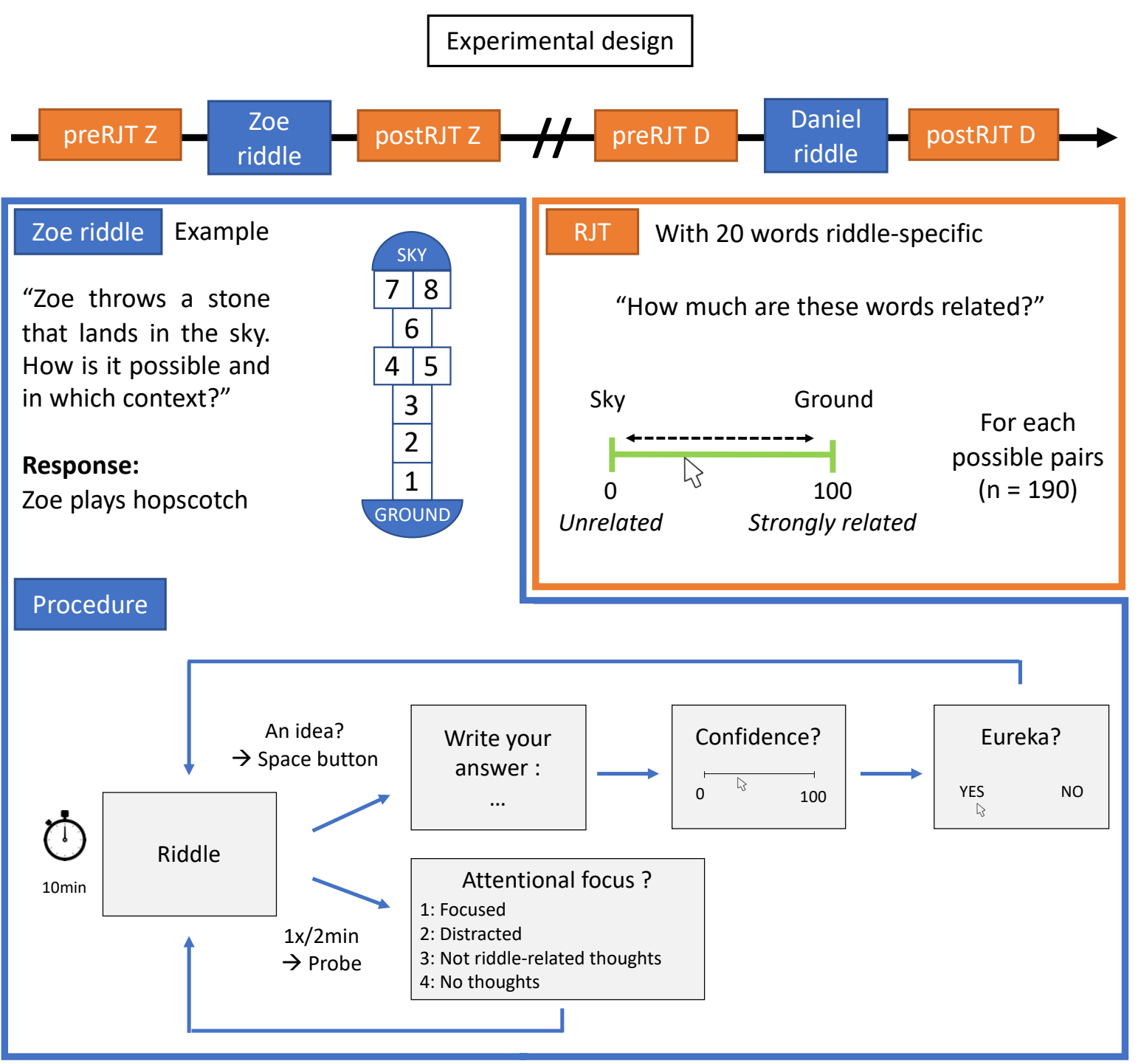


Figure 1. Experimental procedure. Top: Each participant had to solve two different riddles (blue squares, four possible combinations: Zoe/Daniel, Daniel/Zoe, Bar/Car, or Car/Bar) separated by a 15-min break followed by various creativity tasks (not shown in the figure). Each riddle-specific RJT was performed before the problem presentation and after the attempted solving (orange squares). The figure depicts a participant who had to solve first Zoe (Z) and then Daniel (D) riddles. The riddle-specific RJTs were used before and after the Zoe riddle (preRJT Z and post-RJT Z) and the Daniel riddle (pre-RJT D and post-RJT D). Pre and Post-RJT were similar for a given riddle, and a trial is illustrated in the orange square. Blue square: We used the Zoe riddle as an example. Hopscotch is a famous backyard children game in which players must throw a stone into squares drawn on the ground and then hop on them. In France, the last square is traditionally represented by a half-circle and named 'sky'. The experimental procedure is provided in the lower panel. Participants had up to 10 minutes to search for the solution and could propose as many solutions as they wanted during this period. For each answer, they were asked to successively indicate their confidence level about the appropriateness of the proposed solution and whether this solution came to their mind with a subjective experience of Eureka. Every two minutes, we probed them to indicate their attentional focus. Orange square: Participants had to rate the strength of semantic association between two words on a visual scale from 0 ('unrelated') to 100 ('strongly related'). For instance, they had to assess the degree to which the word 'sky' is related to the word 'ground'. Twenty unique words were selected for each riddle and thus differed between RJTs. Word pairs composing riddle-specific RJT trials were all possible combinations of these 20 words $(n=190$ pairs). RJT: Relatedness judgement task.

\section{Solving rate, Eurekas, and attentional focus on the riddle}

The solving rate varied between riddles and was $6.7 \%$ for the Bar $(n=3 / 45$ solvers, 2 with Eureka), $8.2 \%$ for the Daniel ( $n=4 / 49$ solvers, 1 with Eureka), $18.4 \%$ for the Car $(n=9 / 49$ solvers, 3 with Eureka), and $28.6 \%$ for the Zoe $(n=14 / 49$ solvers, 5 with Eureka) riddles. There was no significant difference in solving rate whether a given riddle was performed first or second (Zoe riddle: $\chi^{2}(1)=1.84, p=.18$; Car riddle: $\chi^{2}(1)=4.5910^{-3}, p=.95$; Bar riddle: $\chi^{2}(1)$ $=2.3810^{-30}, p=1$; and Daniel riddle: $\chi^{2}(1)=2.59, p=.11$; all with Yates's continuity correction). For all riddles, $100 \%$ of the solvers found the solution during the 10 -minute thinking phase (before performing the post-RJT). We explored the relationship between problem-solving and the Eureka report using a chi-square dependency test considering all responses together from all participants and riddles. We found that correct responses were more often associated with a Eureka (39.93\%) than incorrect response $\left(21.25 \% ; \chi^{2}(1)=4.65, p=\right.$ $.03)$. Confidence levels of participants' responses were significantly higher for correct responses $($ mean $=75.19, \mathrm{SD}=26.77)$ than for incorrect ones $($ mean $=35.38, \mathrm{SD}=22.42$, $t(214)=8.66, p<.001)$. During the solving phase, the proportion of participants who reported being focused remained above $50 \%$ for each probe, suggesting that participants were mostly engaged in solving the riddle (Figure S1).

Importantly, we explored how the ability to solve our riddles related to creative abilities measured with several tasks including an adaptation of the Remote Associate Task ${ }^{34,35}$, the short version of the Torrance Test of Creative Thinking ${ }^{36}$, and a questionnaire assessing creative activities and achievements ${ }^{37}$ (see supplementary material SI2). We found that creativity scores in all tasks and in the ICAA positively and significantly correlated with the 
solving rate of the four riddles (see supplementary results SI3 and Figure S2). These results indicate that solving our riddles involves creativity-related processes.

Solving success was not associated with a global restructuration of SemNets

We explored whether the global properties of riddle-related SemNets changed as a function of group (solvers or non-solvers) and time (Pre-SemNet and Post-SemNet; Figure 2). As the solving rate for each independent riddle was overall low, we pooled the riddles into pairs (Zoe/Bar and Car/Daniel) in order to maximize the number of solvers while ensuring that a same participant was not included twice in the same analysis (e.g., a participant tested with the Zoe riddle always did the Daniel one; see method). We performed a factorial repeated measures ANOVA to test the main effect of group (solver/non-solvers), time (Pre-SemNet/Post-SemNet), and interaction (group $\mathrm{x}$ time) on each of the global network metrics (Eff $f_{\text {global }}, \mathrm{CC}_{\text {global, }}$, and $\mathrm{Q}_{\text {global }}$ ) and each riddle pair (Zoe/Bar and Car/Daniel). None of the effects were significant, except for the main effect of time on $\operatorname{Eff}_{\text {global }} \mathrm{F}(1,143)=11.56, p<.001, \eta^{2}=.01$, for the couple $\mathrm{Car} /$ Daniel riddle pair. It revealed that SemNet efficiency was higher in the pre-SemNet compared to the post-SemNet. Overall, our results suggest that: i) solvers and non-solvers had a similar organization of the riddle-related semantic associations at baseline (no interaction group $\mathrm{x}$ time); ii) problem-solving was not reflected by SemNet changes at the global level, and iii) the global structure of SemNets remains largely stable over time ${ }^{32}$. 

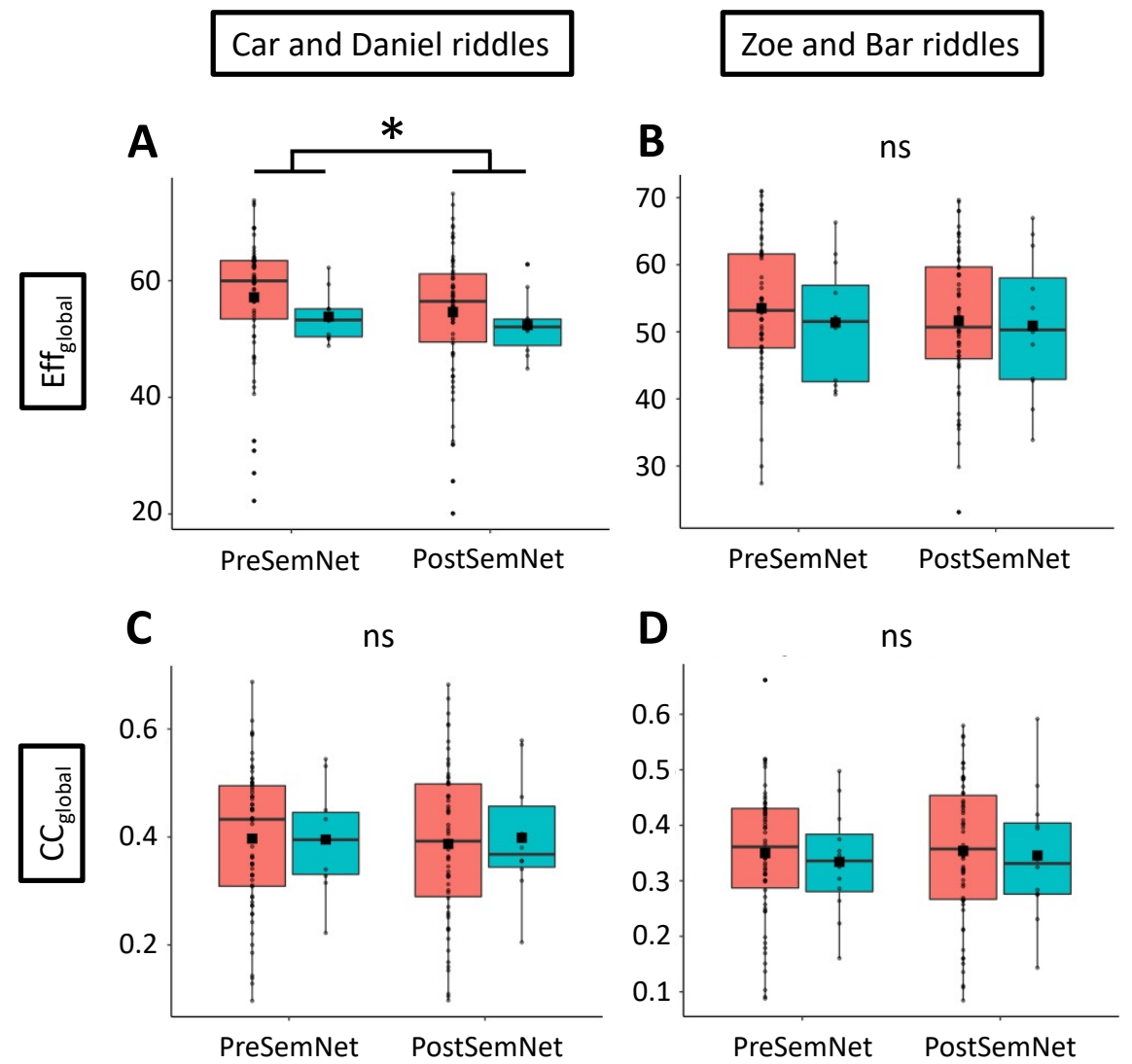

D ns
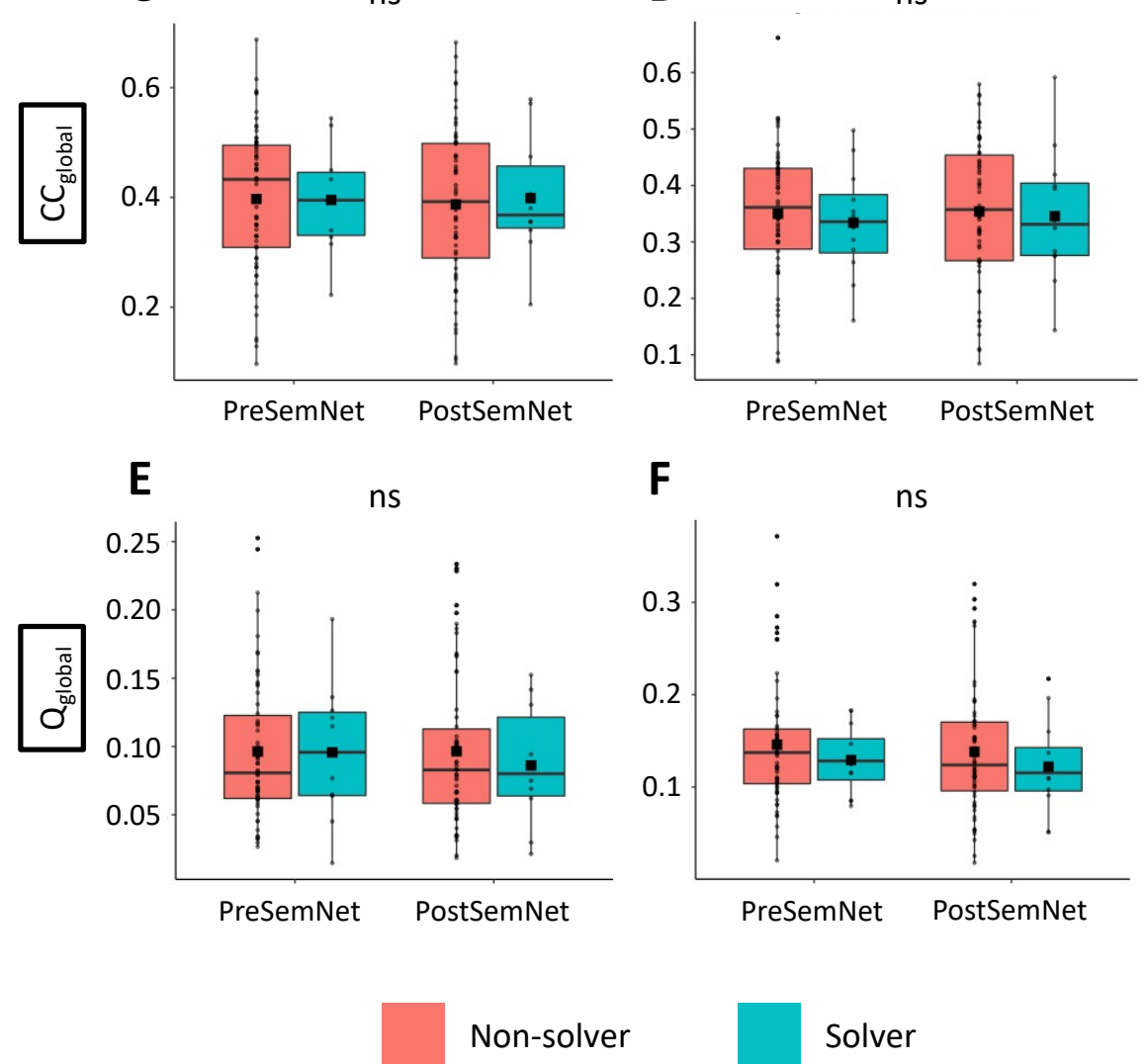

Figure 2. Global SemNet restructuration was not associated with problem-solving. Global metrics of SemNets as a function of the group (solvers in blue and non-solvers in red) and time (Pre-SemNet and Post-SemNet) for efficiency - Eff global (A and B), clustering coefficient $\mathrm{CC}_{\text {global }}(\mathbf{C}$ and $\mathbf{D})$, and modularity - Q global $(\mathbf{E}$ and $\mathbf{F})$. Global metrics were computed within the couple of riddles grouping Car and Daniel $(\mathbf{A}, \mathbf{C}$, and $\mathbf{E})$ and $\operatorname{Zoe}$ and $\operatorname{Bar}(\mathbf{B}, \mathbf{D}$, and $\mathbf{F})$. Each dot represents a subject, color boxes represent the upper and lower quartiles, and black squares indicate the mean. ${ }^{*}: p<.05$, ns: non-significant differences. 
We then explored whether the ability to solve problems could be reflected in local changes in SemNet organization, either at the node or the edge level. We used nonlinear mixed-effects models to predict problem-solving based on the difference between individual-based SemNet metrics over time $\left(\Delta\right.$ Metric $=$ Metric ${ }_{P o s t S e m N e t}-$ Metric $\left.{ }_{P r e S e m N e t}\right)$, the impact ratings (measuring the importance of each node or edge to solve the problem, as assessed by an independent sample), and the interaction between the two (see methods). We added participants as a random effect in each model. Here, we were particularly interested in the interaction effect between the $\Delta$ Metric and the impact ratings, which allowed us to assess whether there was a specific restructuration in the edges (word pairs) or nodes (words) that were the most relevant for solving the problem. We ran a separate model for each local metric computed ( $\mathrm{EV}_{\text {local }}, \mathrm{CC}_{\text {local }}$, $\mathrm{W}_{\text {local }}$ and Eff local $_{\text {). }}$.

Whereas the main effect of $\Delta$ Metric or impact rating alone was not significant for any of the tested metrics (all $p$ 's $>.1$ ), we found a significant interaction effect of $\Delta$ Metric and impact rating on problem-solving for all SemNet metrics: $\mathrm{W}_{\text {local }}, \boldsymbol{\beta}=7.0810^{-2}, p=.01$; Eff local, $_{\text {, }}$ $\boldsymbol{\beta}=8.0810^{-2}, p=.004$; and $\mathrm{EV}_{\text {local }}, \boldsymbol{\beta}=0.28, p=.002$ (Figure 3), except for $\mathrm{CC}_{\text {local }}, \boldsymbol{\beta}=2.63$ $10^{-2}, p=0.88$ (Table S4). This interaction effect indicates that larger positive changes in $\mathrm{W}_{\text {local }}$, $\mathrm{Eff}_{\text {local, }}$, and $\mathrm{EV}_{\text {local }}$ in pairs/words judged as more helpful to solve the riddles predicted higher solving success. In other words, helpful word-pairs were rated in the post-RJT as more related and connected ( $\mathrm{W}_{\text {local }}$ and $\left.\mathrm{Eff}_{\text {local }}\right)$ after successfully solving the problem than before the problem presentation, and helpful nodes became more central/influential in the networks $\left(\mathrm{EV}_{\text {local }}\right)$. Note that the balanced accuracies of the three significant models ranged between .70 and .79 , indicating that it is a powerful manner to predict problem-solving. These findings are consistent with the hypothesis that solving success implies a local restructuration of SemNet targeting the most helpful nodes and edges ${ }^{10}$.

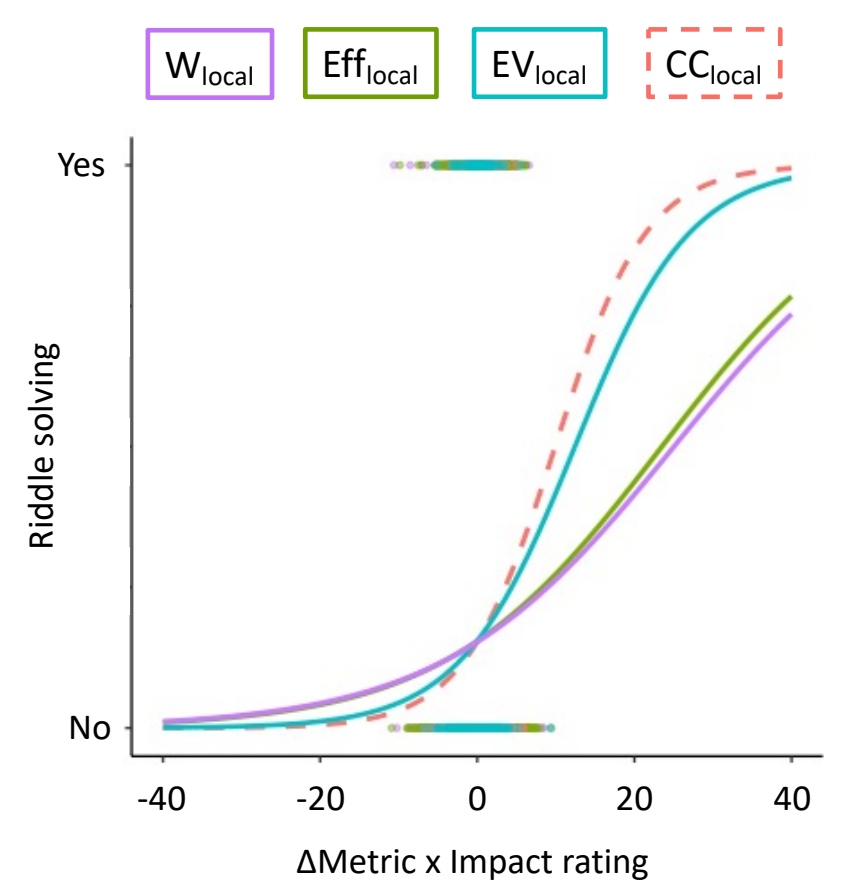

Figure 3. Local SemNet restructuration was associated with problem-solving. Lines represent the fitting curves of the interaction effect ( $\Delta$ Metric x Impact rating) in the four mixed models computed for local SemNet metrics (weight $-\mathrm{W}_{\text {local }}$ in purple, efficiency - Eff local in green, eigenvector centrality $-\mathrm{EV}_{\text {local }}$ in blue, and clustering coefficient $-\mathrm{CC}_{\text {local }}$ in red). Each 
dot represents an individual with the same color code, with the $\Delta$ Metric weighted by the impact 293 ratings ( $\Delta$ Metric $\mathrm{x}$ Impact rating) on the $\mathrm{x}$-axis and solving success on the $\mathrm{y}$-axis. A solid line indicates that a significant effect $(p<.05)$ was found.

An illustration of the results is provided for the Zoe riddle (riddle with the highest solving rate). In this riddle, participants had to explain a situation in which "Zoe throws a stone
that lands in the sky". The solution is that Zoe is playing hopscotch (a famous backyard children's game in which the players must throw a stone into squares drawn on the ground and then hop on them). In France, the last square is traditionally represented by a half-circle and named 'sky' (Figure 1). Hence, to find the solution, one needs to represent the sky in another way (i.e., a drawing on the floor) than the usual one (i.e., the place where birds and planes fly). SemNets at the individual level are provided with examples of one solver and one non-solver (Figure 4, see Figure S3 to see SemNets at the group level by averaging the $\Delta$ Metrics within the solver and non-solver group). We can observe that more changes occurred in the solvers' SemNet than the non-solvers one, suggesting a higher restructuration related to solving. Additionally, positive changes were mainly observed in helpful nodes and edges in the solvers' network. 

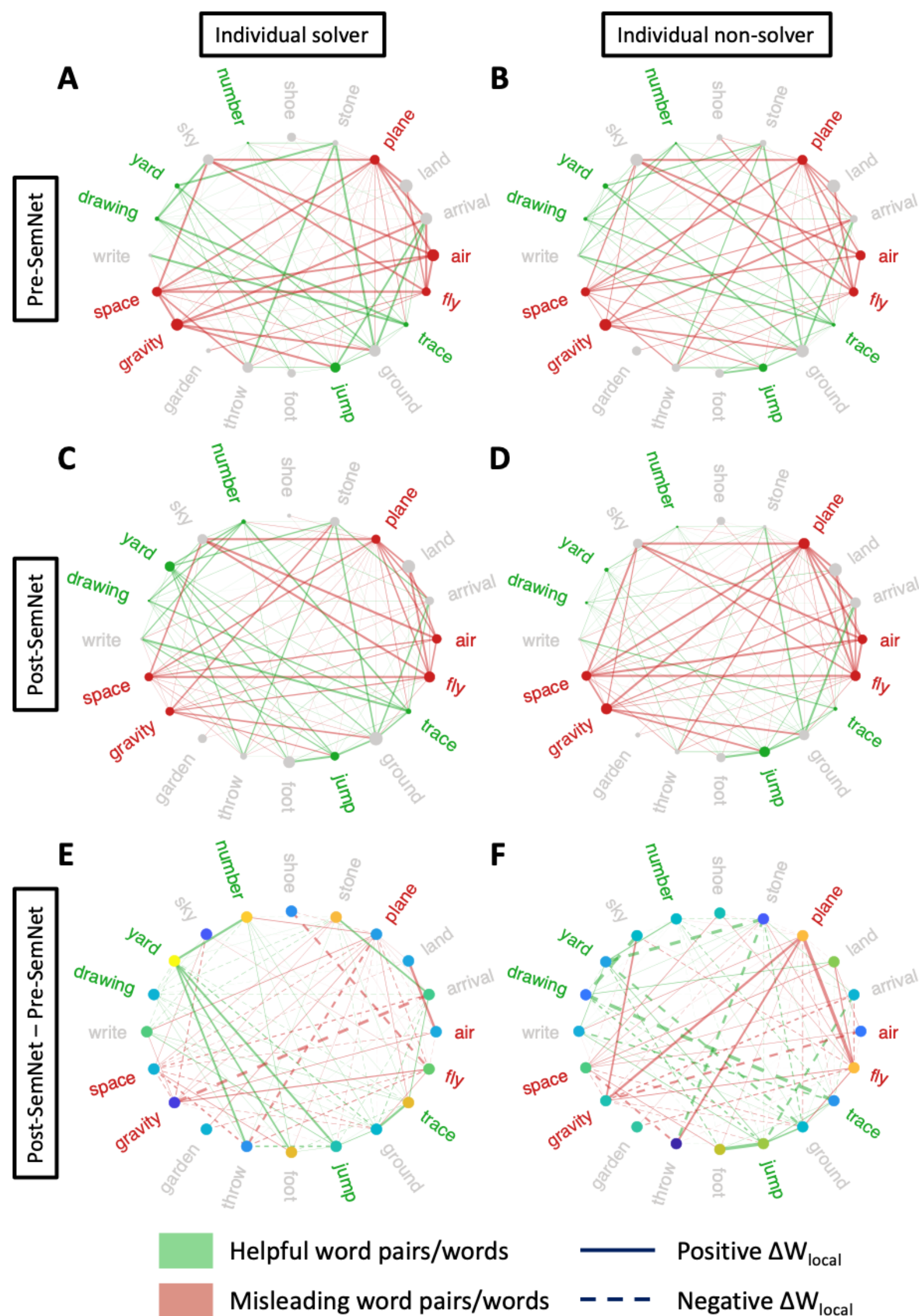

Helpful word pairs/words

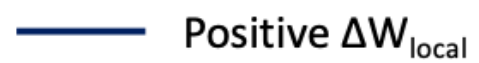

Misleading word pairs/words - - Negative $\Delta \mathrm{W}_{\text {local }}$

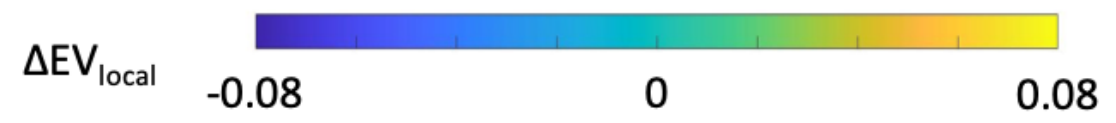



solver. Top: SemNets based on the RJT performed before the presentation of Zoe riddle (PreSemNet) for one individual who solved the problem (A) and one who did not (B). Middle: SemNets based on the RJT performed after working on the riddle (Post-SemNet) for the same individuals (C: solver, D: non-solver participant). Bottom: Difference between the two SemNets (Post-SemNet - Pre-SemNet) for the same individuals (E: solver, F: non-solver). For all SemNets, a circle layout is used so that the nodes keep the same position. We categorized the pairs and words as helpful or misleading based on the distribution of the impact ratings (Figure S4 and S5): scores of pairs/words in the first quartile were labeled as misleading $(\mathrm{n}=$ 48 pairs and $\mathrm{n}=5$ nodes respectively) whereas those in the last quartile were labeled as helpful ( $\mathrm{n}=48$ pairs and $\mathrm{n}=5$ nodes respectively). Node colors represent words that have been categorized as helpful (in green) or misleading (in red) to solve the problem. Only helpful and misleading pairs are represented as edges (respectively in green and red). In $\mathbf{A}$ to $\mathbf{D}$, node size is proportional to the $\mathrm{EV}_{\text {local }}$ value (the bigger the dot, the higher $\mathrm{EV}_{\text {local }}$ ), and edge thickness is proportional to $\mathrm{W}_{\text {local }}$ (the wider the line, the higher $\mathrm{W}_{\text {local }}$ ). In $\mathbf{E}$ and $\mathbf{F}$, node colors represent

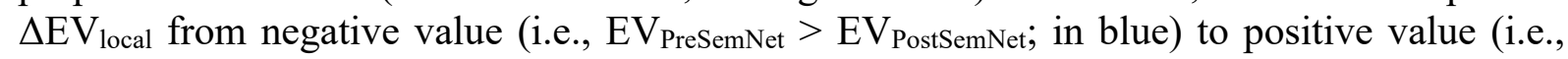
$\mathrm{EV}_{\text {PostSemNet }}>\mathrm{EV}_{\text {PreSemNet; }}$ in yellow), and edge thickness is proportional to the absolute value of $\Delta \mathrm{W}_{\text {local. }}$. Dashed lines represent negative $\Delta \mathrm{W}_{\text {local }}$ (i.e., $\mathrm{W}_{\text {PreSemNet }}>\mathrm{W}_{\text {PostSemNet }}$, whereas solid ones represent positive $\Delta \mathrm{W}_{\text {local }}$ (i.e., $\mathrm{W}_{\text {PostSemNet }}>\mathrm{W}_{\text {PreSemNet }}$ ).

\section{Discussion}

In this study, we explored how restructuring of SemNets - reflecting a reorganization of the mental representation of the riddles - serves as a cognitive mechanism underlying problemsolving. By building individual SemNets before and after solving a problem, we characterized and quantified the changes in the organization of semantic associations predicting successful problem-solving. This method further allowed us to show that the restructuring of SemNets associated with problem-solving is not global, but rather restricted to parts of the networks that are the most relevant to the problem solution. These results shed new light on mental restructuring associated with problem-solving and provide a new method to quantify this restructuring based on SemNets.

Previous research has demonstrated how computational network science methodologies allow exploring the structure of semantic memory and how concepts are organized in individual minds via SemNets ${ }^{16,19-21}$. Recently, investigating semantic memory structure using SemNet has been shown as a tool to empirically explore individual differences in creative abilities and behavior ${ }^{24-26,28-30}$. Here, we demonstrate that in addition to offering a way to investigate how the organization of semantic associations differs in less and more creative individuals, SemNet methods can further be used to explore creative problem-solving, that is, to provide a mechanistic explanation of how a given individual solves problems that he/she never encountered before.

Our results provide empirical support for an oft-cited theory in the creativity literature, which postulates that the restructuration of semantic associations pertaining to a given problem predicts the successful solving of this problem ${ }^{3-5}$. Such restructuration idea has often been associated with the notion of insight ${ }^{38-40}$, i.e., when the solution to an unsolved problem comes suddenly and effortlessly 6,8,11,41. A seminal theory has proposed that cognitive insight may entail the forging or changing of nodes and/or edges in the semantic (small world) network ${ }^{10}$. Such changes supposedly create a 'shortcut' in the network that allows decreasing the distance between different representations and results in a cascade of new connections. Here, we found that solving success was linked with a local increase in efficiency in the edges that are critical 
for the solution, substantiating Schilling's hypothesis of a fast decrease in path length within 363 individual's network representations ${ }^{10}$. However, further work is needed to fully test this 364 theory. The low number of solvers in our study prevented us from directly exploring the effect of insight reports on the restructuration of semantic associations.

To provide a quantitative measure of restructuring, we applied here an original SemNet approach at the individual level. Our work complements and extends a pioneering study ${ }^{12}$, which used a pathfinder scaling algorithm to show at the group level that the SemNet of participants who solved the Bar riddle was different from the SemNet of participants who did not solve the riddle, and from the SemNet of those who were given the solution. Our work extends these results by several means. First, we estimated SemNets at the individual level, using the RJT procedure ${ }^{25}$, which allowed us to consider inter-individual variability in semantic associations. Additionally, instead of examining only the structure of SemNet after working on the riddle, we explored individual changes within the SemNet. It allowed us to consider changes of SemNet local properties as a marker of restructuring. Second, we characterized the restructuration predicting problem-solving as changes in several specific SemNet metrics (weight, efficiency, and eigenvalue centrality) that provide valuable information about how individual SemNets are structured. Importantly, our approach allows demonstrating that a problem-related restructuration occurs at a local level, in edges and nodes that were independently assessed as important to the riddle's solution. Interestingly, we did not find any significant differences in the global structure of SemNets between solvers and non-solvers, indicating that networks are quite stable across participants or that the analyses performed at the group level might be less efficient in capturing subtle differences (see also ${ }^{32}$ ). Finally, participants worked on four different riddles instead of one riddle in the Durso et al. ${ }^{12}$ study. Including the four riddles in our nonlinear mixed models provides evidence that the results generalize across riddles and are not dependent on the tested riddle. In addition, we provided strong evidence of the relationship between the ability to solve our four riddles and other creativity abilities. It suggests that our riddles capture some aspects of creative ability and can be considered as creative problem-solving. By adding participants as a random effect in our models, we took into account inter-individual variability in predicting problem-solving, which allowed us to focus on the SemNet changes as a mechanism of problem-solving rather than as an individual capacity.

Some limitations must be mentioned. First, one might argue that some word pairs proposed during the RJT primed the riddle's solution and gave participants hints. This is unlikely since only a few participants found the solution, and all of them found it before the post-RJT. Second, the solving rate of the riddle was overall low. It was especially the case for the Bar riddle, whose solving rate was inferior to the one observed in the Durso et al. study ${ }^{12}$. It may be because participants had only 10 minutes to think of a solution (vs. 2 hours in Durso et al. study ${ }^{12}$ ). Of note, in contrast to this previous study, we did not allow participants to ask questions, thus limiting inter-individual differences in the quantity of information we provided to each of them. Besides, the low solver rates did not allow us to explore the restructuration of semantic associations at the riddle level. Further studies with a larger variety of riddles and including a control riddle that does not require restructuration would be helpful to conclude on the specificity of SemNet restructuration for creative problem-solving. Finally, a critical question that our findings leave open relates to the direction and causal link between restructuring and solving. Does the restructuration we observed in SemNet indeed lead to solving the riddle? Or does finding the riddle solution change the relatedness judgments during the post-RJT? The current study design does not allow us to conclude on this question. However, we provide a new method to explore in further studies the neural correlates of restructuring that can contribute to address the opened question about its causality with problem-solving. 
To conclude, we directly examined the restructuring effects of semantic memory that

\section{Methods}

\section{Participants}

Ninety-nine native French speakers aged between 18 and 38 years old (mean age $=25.91$ years, $\mathrm{SD}=4.74 ; 69$ women) were included. All participants were healthy adults with no history of neurological and/or psychiatric illness and no psychoactive substance abuse. An approved ethics committee approved the study. All the participants gave their written informed consent and received $10 €$ /hour as financial compensation.

\section{Experimental procedure}

Participants underwent a $4 \mathrm{~h}$-experiment during which they had to solve two riddles. They were first presented with one riddle and attempted to solve it during 10-min. Before and after this solving phase, participants performed the RJT task. After a 15-min break followed by various creativity tasks, participants were presented with a second riddle, following the exact same procedure as for the first one (RJT before and after a 10-min solving phase). All tasks were computed using the Psychopy software ${ }^{45}$ running on individual computers in a classroom dedicated to cognitive experiments (https://institutducerveau-icm.org/fr/prisme-humanbehavior-exploration-core-facility/). Task-related instructions were initially explained and repeated before the beginning of each task. During the $4 \mathrm{~h}$-experiment, participants performed additional separate tasks that are out the scope of this study.

\section{Problem-solving phase}

After each riddle presentation, participants had up to 10 minutes to search for the solution (Figure 1, blue square). During this time, the riddle remained displayed at the center of the screen. Participants were instructed to report all the ideas that came to mind, even if they judged them bizarre or irrelevant. They could press the space button anytime to propose a response but had to do it only if they knew what to write (to avoid getting additional thinking time). Pressing the space bar stopped the timer. Participants then had up to 30 seconds to write their ideas using the keyboard. The number of ideas that a participant could propose was not limited, and no feedback on the response correctness was provided. For each response they gave, the participants were first asked to indicate the confidence they had in their response on a visual scale from 0 ("not sure at all") to 100 ("completely sure"). Afterward, a new screen displayed 
"Eureka?", and the participants indicated whether their response came to their mind with an insight or Eureka phenomenon by pressing a "yes" or "no" button ${ }^{46}$. Participants were told that a Eureka is "the subjective experience you may have when you solve a problem and the solution comes to mind suddenly, is not the direct result of cognitive efforts, and you are not able to report the mental steps leading to this solution". It was opposed to analytic solving where "you have a strategy and the feeling of gradually getting closer to the solution". Participants were also explained that these two solving methods were not exclusive and instructed the participants to consider only the few seconds before the idea came to their mind. Once the participants replied to the Eureka question, the riddle was displayed again, and the timer restarted. Every two minutes, we probed the participants' attentional focus by asking them what they were thinking about. The participants answered the question by choosing between four options (" Focused on the riddle », "Distracted by the environment", "Thoughts unrelated to the riddle », « No thoughts ») using the keyboard (a predetermined numerical key corresponded to each option).

We counterbalanced the order of the riddles within the experimental design to ensure that neither positive (training) nor negative (fatigue) effects related to which riddle came first could interfere with the results. Hence, half of the participants worked on one set of riddles (Zoe/Daniel riddles), and another half worked on another set of riddles (Car/Bar riddles). The order of the riddle presentation in each set was counterbalanced among participants.

\section{Relatedness Judgment Task}

During the RJT, participants were presented with all possible combinations of word pairs ( $\mathrm{n}=$ 190) based on a riddle-specific 20 -word list. On each trial, a different word pair was displayed on the screen, and participants were asked to rate the strength of semantic association or relatedness between the two words on a visual scale from 0 ("unrelated") to 100 ("strongly related"), using a slider (Figure 1, orange square). The visual scale was displayed below the word pair on each trial and stayed on the screen until the participant responded. Participants had up to 4.5 seconds to respond using the computer mouse and validate their rating with a leftclick. The order of trials was initially pseudo-randomized and then fixed across subjects. We used the Mix software ${ }^{47}$ to generate a pseudo-random order where each word appeared equally on the right and left sides of the screen and did not repeat in two consecutive trials. Before starting the task, the participants were instructed to answer as quickly and spontaneously as possible and completed 25 practice trials. Before each first riddle-specific RJT, all the words that composed the pairs were successively displayed on the screen.

\section{Materials}

\section{Riddle construction}

To test whether creative problem-solving is related to a restructuring of semantic associations, we required to construct problems that: i) were verbal (i.e., to relate to the RJT method used to allow building SemNet); ii) had a unique solution (i.e., to facilitate the classification of participants as solvers or non-solvers); iii) were difficult enough (i.e., to be able to measure a change after a solving phase); and iv) were likely to necessitate creative thinking (i.e., to maximize our chances to observe a restructuring of semantic associations). Problems requiring creative thinking are often ill-defined (i.e., no specific heuristics or usual rules could be used to solve the problem) and putatively prompt a usual representation that one needs to overcome in order to solve the problem. Riddle like the one found in Durso et al. ${ }^{12}$, satisfies all of those 
criteria. We thus selected the Durso riddle and translated it into French (the "Bar" riddle). As we could not find any other similar riddles in the scientific literature, we picked one relevant riddle displayed on different riddle websites and rated as tricky (the "Car" riddle). Finally, we created two novel riddles in such a way that the problem situation/solution was analogous to the "Bar" riddle (the "Daniel" riddle; in both riddles, a person with a threatening behavior is actually helpful) and to the "Car' riddle (the "Zoe" riddle; in both riddles, a person apparently involved in a real-life situation is actually playing a game). English-translated riddles are available in Tables $\mathbf{S} \mathbf{1}$ and $\mathbf{S 2}$.

\section{Riddle-specific RJT material}

We built a verbal material for the RJT task that was specific to each riddle separately and consisted of a list of 20 different words (four different lists for Zoe, Daniel, Car, and Bar riddles; Table S3). The same list was used in the RJT performed before (pre-RJT) and after (post-RJT) working on a given riddle. To create those lists, we used a two-step procedure (see supplementary material). Briefly, in each word list, we selected words semantically related to the riddle (in average $4+/-.80$ words across the riddles) or its solution (mean $=6+/-1$ words) and words unrelated to it (mean $=10+/-.10$ words). Words in the lists are either verbs, nouns or adjectives. We chose frequent words defined as lexical frequency higher than one million occurrences using the lexicon database (http://www.lexique.org/; ${ }^{48}$ ).

To ensure that our newly-created RJT lists could capture those associations that are critical for solving the riddles, we asked nine independent and naive judges to rate how helpful or misleading the link between the words of each of the 190 pairs was in solving a given riddle. The judges scored each riddle separately. They first read the riddle and were given the solution. Then, they rated the importance of each word pair to solve the riddle using a visual scale ranging from -50 (« misleading ») to 50 (« helpful »), centered on 0 ("neutral"). Each word pair was successively displayed on the screen above the visual scale in the same way as during the RJT procedure described above. There was no time limit to respond. By averaging the ratings across the judges and then z-scoring across the 190 word pairs, we obtained an 'impact rating', which quantifies the relevance of each word pair $(n=190)$ for solving the riddle. We also computed an indirect impact score determining the importance of each word to solve the riddle. This indirect score was calculated by averaging the impact rating of the pairs involving this word ( $\mathrm{n}$ $=19$ pairs per word) for each word. Then, we z-scored the obtained values across the 20 riddlespecific words. This procedure was repeated for each riddle-specific word list. These impact ratings allowed us to weight word pairs (edges) and words (nodes) according to their relevance for solving the problem: the higher the score, the more we considered the pair or the word helpful.

The distribution of impact ratings for both word pairs and words alone are provided in Figure S4 and S5, respectively. Intraclass coefficient (ICC) was higher than .80 for all riddles (Zoe: ICC $=.93$; Car: ICC $=.84$; Bar: ICC $=.90$; and Daniel: ICC $=.94)$, suggesting a good inter-judge reliability. Distributions of impact ratings show that our riddle-specific RJT material captures word associations of varying degrees of relevance to solving the riddle.

\section{Behavioral analyses of the solving phase}

For each riddle, participants were assigned to either the solver or non-solver group, depending on their success in solving the corresponding riddle. We computed the solving rate as the percent of participants who gave the correct solution anytime during the time allowed, i.e., the number of solvers divided by the number of participants who worked on this riddle, excluding participants who knew the riddle beforehand. Of note, six participants were excluded because 
they were already familiar with the riddle $(\operatorname{Bar}, \mathrm{n}=5$; Car, $\mathrm{n}=1)$. Among the solvers, we indicated the proportion of participants who reported a Eureka feeling ${ }^{8,46}$. For each riddle separately, we tested if the riddle's order in the design impacted solving success using a chisquare analysis (corrected with Yates method if needed). Then, we explored the relationships between solving success and Eureka report with a chi-square test using all the riddles and all the participants pooled together excluding missing values listwise. In addition, we compared the average confidence across participants when a response was correct (if there is one) vs. when responses were incorrect (that is, the average confidence of all incorrect responses at the individual level) for all riddles together using two-tailed paired-samples $t$-test analyses.

\section{Semantic network analysis}

\section{Semantic network estimation}

For each participant and each riddle independently, we built two SemNets based on RJT ratings collected before the presentation of the problem (Pre-SemNet) and after attempting to solve the problem (Post-SemNet) (Figure 1). Based on previous studies using this approach ${ }^{25,26,29,30}$, we used weighted undirected network method (WUN), in which the nodes were the preselected words and the edges represented the ratings during the RJT. In undirected networks, the relation between node $a$ and node $b$ is equal to the relation between node $b$ and node $a$, which conforms to the nature of the judgment task. In WUN, all links between the words (nodes) are weighted, here using the individual RJT ratings.

For each WUN SemNet (pre-RJT and post-RJT), we computed SemNet metrics that quantify the connectivity properties of a network using the Brain Connectivity Toolbox ${ }^{33}$ (version 2019-03-03) running with Matlab R2020. We excluded from SemNet metrics analyses participants who did not respond or rated the word pair as zero in more than $10 \%$ of all trials in one RJT. We considered that these participants were not sufficiently engaged in the RJT and that missing or zero ratings would importantly bias the network with missing links. Nonresponses have been substituted by a zero for metrics cannot be computed if missing values are included.

First, we explored the global topology of the networks using global SemNet metrics, that have been commonly used in cognitive research ${ }^{16,20,33,49,50}$. These metrics include (i) the efficiency (Eff global, averaged inverse shortest path length in the network), (ii) the clustering coefficient ( $\mathrm{CC}_{\text {global }}$, degree to which neighbor nodes are also connected to one another), and (iii) the modularity (Qglobal, degree to which the network is segregated). Second, we aim to use SemNets to study focal restructuration effects. Thus, we computed local SemNet metrics focusing on each node $(n=20)$ and edge $(n=190)$. Specifically, we computed several metrics that reflect local connectivity properties and estimate how central or efficient a node or an edge is. At the node level, we computed the eigenvector centrality ( $\mathrm{EV}_{\text {local, }}$ self-referential measure of centrality where nodes have high EV if they connect to other nodes with high EV) and the clustering coefficient $\left(\mathrm{CC}_{\text {local }}\right)$. We chose $\mathrm{EV}$ as the centrality measure, based on a previous study that showed that EV has higher reliability compared to other centrality measures in cognitive networks ${ }^{51}$. At the edge level, we used the weights $\left(\mathrm{W}_{\text {local }}\right)$ and computed the efficiency $\left(\mathrm{Eff}_{\text {local }}\right)$. Then, we compared pre- and post-SemNets local metrics to explore changes in the properties of SemNets that could be related to creative problem-solving. For this purpose, we calculated an individual difference for each metric between the SemNet built after and before a riddle $\left(\Delta\right.$ Metric $=$ Metric Post-SemNet - Metric $\left._{\text {Pre-SemNet }}\right)$. The higher the $\Delta$ Metric, the larger the increase in the considered metric after working on the riddle.

\section{Statistical analyses}


To explore whether a restructuration of SemNet can be measured at the global level and related to problem-solving, we computed the averaged value of each global metric in the two groups (solvers and non-solvers) at the two different times (Pre-SemNet and Post-SemNet). As the solving rate for each independent riddle was overall low, we pooled the riddles two by two (Zoe/Bar and Car/Daniel) in order to maximize the number of solvers while ensuring that a same participant was not included twice in the same analysis (e.g., a participant who was tested with the Zoe riddle always also performed the Daniel riddle). We ran a factorial ANOVA with repeated measures, with successful solving as an inter-subject factor and time as an intra-subject factor. We repeated this analysis for each global metric $\left(\mathrm{Eff}_{\text {global }}, \mathrm{CC}_{\text {global }}\right.$, and $\left.\mathrm{Qglobal}\right)$ and each riddle couple (Zoe/Bar and Car/Daniel).

Then, we used a nonlinear mixed-effects model to explore how local changes in SemNets could predict problem-solving. In these models, successful solving was the dependent variable (isSolved, binary variable), and the fixed-effect predictors were the difference between the two SemNets of a given local metric ( $\Delta$ Metric, continuous variable), the impact rating (Impact rating, continuous variable), and the interaction factor between the two ( $\Delta$ Metric $\mathrm{x}$ Impact rating, continuous variable). $\Delta$ Metric and Impact rating variables were $z$-scored. Participants were entered as a random-effect factor in the model. It allows us to take into account the repeated measures across subjects as a random-effect factor (Subject, maximum two riddles per subject) and inter-individual variability. The model can be formalized as follows:

$$
\begin{gathered}
\text { isSolved }=\boldsymbol{\beta}_{1} \mathrm{x} \Delta \text { Metric }+\boldsymbol{\beta}_{2} \mathrm{xImpactRating}+\boldsymbol{\beta}_{3} \mathrm{x}(\Delta \text { Metric } \mathrm{x} \text { Impact rating }) \\
+(1 \mid \text { Participant })+(-1+\Delta \text { Metric } \mid \text { Subject })+\mathrm{k}
\end{gathered}
$$

We replicated the same model for each local metric $\left(\mathrm{W}_{\text {local }}, \mathrm{Eff}_{\text {local }}, \mathrm{CC}_{\text {local }}\right.$, and $\left.\mathrm{EV}_{\text {local }}\right)$. All nonlinear mixed model were run on Rstudio (v 1.4.1717) with glmer function. For each significant model, we calculated the balanced accuracy. It measures the probability that the model correctly classifies the variables according to the condition to be explained (i.e., solver or non-solver), considering its unbalanced distribution.

\section{Acknowledgements}

This research was partly funded by the 'Agence Nationale de la Recherche' [grant number ANR-19-CE37-001-01], the 'Fondation pour la recherche medicale' [grant number DEQ20150331725], and from the program "Investissements d'avenir" [grant number ANR-10IAIHU-06]. TB also received funding from the 'Assistance Publique des Hôpitaux de Paris'. MOT is funded by Becas-Chile of ANID (CONICYT). CL is funded by the Doctoral school ED3C and 'Société Française de Recherche et Médecine du Sommeil' (SFRMS). Part of this work was carried out in the PRISME facility of ICM. We gratefully acknowledge Karim N'diaye for his help in the data collection. We thank all the participants to the study and Clothilde Chappé for piloting part of the material used in this study.

\section{Authors' contribution}

Conceptualization: YK, DO, EV; Methodology: TB, YK, MOT, ALP, CL, DO, EV; Investigation: TB; Visualization: TB; Supervision: DO, EV; Writing: TB, YK, DO, EV. 
663

The authors declare that they have no competing conflict of interests.

665

666

667

668

669 The material, the data sets generated and/or analyzed during the current study, and the scripts 670 written will be available in a persistent repository. Data sharing will be anonymized and will 671 not include participants' demographical information. 
1. Newell, A. \& Simon, H. A. Human problem solving. vol. 104 (Prentice-hall Englewood Cliffs, NJ, 1972).

676 2. Reitman, W. R., Grove, R. B. \& Shoup, R. G. Argus: An information-processing model 677 of thinking. Behav. Sci. 9, 270 (1964).

678 3. Mumford, M. D., Mobley, M. I., Reiter-Palmon, R., Uhlman, C. E. \& Doares, L. M. 679 Process analytic models of creative capacities. Creat. Res. J. 4, 91-122 (1991).

680 4. Finke, R. A., Ward, T. B. \& Smith, S. M. Creative cognition: Theory, research, and 681 applications. (1992).

682 5. Cropley, A. In praise of convergent thinking. Creat. Res. J. 18, 391-404 (2006).

683 6. Topolinski, S. \& Reber, R. Gaining Insight Into the "Aha" Experience. Curr. Dir. 684 Psychol. Sci. 19, 402-405 (2010).

685 7. Chu, Y. \& MacGregor, J. N. Human performance on insight problem solving: A review. 686 J. Probl. Solving 3, 6 (2011).

687 8. Kounios, J. \& Beeman, M. The cognitive neuroscience of insight. Annu. Rev. Psychol. $688 \quad \mathbf{6 5}, 71-93(2014)$.

689 9. Mednick, S. A. The associative basis of the creative process. Psychol. Rev. 69, 220-232 690 (1962).

691 10. Schilling, M. A. A 'small-world' network model of cognitive insight. Creat. Res. J. 17, 692 131-154 (2005).

693 11. Ohlsson, S. Restructuring revisited: II. An information processing theory of 694 restructuring and insight. Scand. J. Psychol. 25, 117-129 (1984).

695 12. Durso, F. T., Rea, C. B. \& Dayton, T. Graph-Theoretic Confirmation of Restructuring 696 During Insight. Psychol. Sci. 5, 94-98 (1994).

697 13. Danek, A. H., Williams, J. \& Wiley, J. Closing the gap: connecting sudden 698 representational change to the subjective Aha! experience in insightful problem solving. 699 Psychol. Res. 84, 111-119(2020).

700 14. Öllinger, M., Jones, G., Faber, A. H. \& Knoblich, G. Cognitive mechanisms of insight: 701 The role of heuristics and representational change in solving the eight-coin problem. J. Exp. 702 Psychol. Learn. Mem. Cogn. 39, 931-939 (2013).

703 15. Ash, I. K., Cushen, P. J. \& Wiley, J. Obstacles in investigating the role of restructuring 704 in insightful problem solving. J. Probl. Solving 2, (2009).

705 16. Siew, C. S., Wulff, D. U., Beckage, N. M. \& Kenett, Y. N. Cognitive network science: 706 A review of research on cognition through the lens of network representations, processes, and 707 dynamics. Complexity 2019, (2019).

708 17. Hills, T. T. \& Kenett, Y. N. Networks of the Mind: How Can Network Science Elucidate 709 Our Understanding of Cognition? Top. Cogn. Sci. (2021).

710 18. Steyvers, M. \& Tenenbaum, J. B. The large-scale structure of semantic networks: 711 Statistical analyses and a model of semantic growth. Cogn. Sci. 29, 41-78 (2005).

712 19. Baronchelli, A., Ferrer-i-Cancho, R., Pastor-Satorras, R., Chater, N. \& Christiansen, M. 713 H. Networks in Cognitive Science. Trends Cogn. Sci. 17, 348-360 (2013).

714 20. Borge-Holthoefer, J. \& Arenas, A. Semantic networks: Structure and dynamics. 715 Entropy 12, 1264-1302 (2010).

716 21. Kumar, A. A. Semantic memory: A review of methods, models, and current challenges. 717 Psychon. Bull. Rev. 28, 40-80 (2021).

718 22. Collins, A. M. \& Loftus, E. F. A spreading-activation theory of semantic processing. 719 Psychol. Rev. 82, 407 (1975).

720 23. Kenett, Y. N., Kenett, D. Y., Ben-Jacob, E. \& Faust, M. Global and Local Features of 721 Semantic Networks: Evidence from the Hebrew Mental Lexicon. PLoS ONE 6, e23912 (2011). 
24. Kenett, Y. N., Anaki, D. \& Faust, M. Investigating the structure of semantic networks in low and high creative persons. Front. Hum. Neurosci. 8, (2014). 25. Benedek, M. et al. How semantic memory structure and intelligence contribute to creative thought: a network science approach. Think. Reason. 23, 158-183 (2017). 26. Bernard, M., Kenett, Y. N., Tellez, M. O., Benedek, M. \& Volle, E. Building individual semantic networks and exploring their relationships with creativity. in 138-144 (2019). creativity? Curr. Opin. Behav. Sci. 27, 11-16 (2019).

28. Kenett, Y. N. \& Faust, M. A Semantic Network Cartography of the Creative Mind. Trends Cogn. Sci. (2019) doi:10.1016/j.tics.2019.01.007.

29. He, L. et al. The relation between semantic memory structure, associative abilities, and verbal and figural creativity. Think. Reason. 27, 268-293 (2021).

30. Ovando-Tellez, M. et al. Brain connectivity-based prediction of real-life creativity is mediated by semantic memory structure. bioRxiv (2021).

31. Yee, E. \& Thompson-Schill, S. L. Putting concepts into context. Psychon. Bull. Rev. 23, 1015-1027 (2016).

32. Kenett, Y. \& Thompson-Schill, S. L. Novel conceptual combination can dynamically reconfigure semantic memory networks. (2020).

33. Rubinov, M. \& Sporns, O. Complex network measures of brain connectivity: Uses and interpretations. NeuroImage 52, 1059-1069 (2010).

34. Bendetowicz, D., Urbanski, M., Aichelburg, C., Levy, R. \& Volle, E. Brain morphometry predicts individual creative potential and the ability to combine remote ideas. Cortex 86, 216-229 (2017).

35. Bendetowicz, D. et al. Two critical brain networks for generation and combination of remote associations. Brain 141, 217-233 (2018).

36. Torrance, E. P. Predictive Validity of the Torrance Tests of Creative Thinking*. $J$. Creat. Behav. 6, 236-262 (1972).

37. Diedrich, J. et al. Assessment of real-life creativity: The Inventory of Creative Activities and Achievements (ICAA). Psychol. Aesthet. Creat. Arts 12, 304 (2018).

38. Seifert, C. M., Meyer, D. E., Davidson, N., Patalano, A. L. \& Yaniv, I. Demystification of cognitive insight: Opportunistic assimilation and the prepared-mind hypothesis. (1994).

39. Sprugnoli, G. et al. Neural correlates of Eureka moment. Intelligence 62, 99-118 (2017).

40. Sandkühler, S. \& Bhattacharya, J. Deconstructing Insight: EEG Correlates of Insightful Problem Solving. PLOS ONE 3, e1459 (2008).

41. Ohlsson, S. Information-processing explanations of insight and related phenomena. Adv. Psychol. Think. 1, 1-44 (1992).

42. Cai, D. J., Mednick, S. A., Harrison, E. M., Kanady, J. C. \& Mednick, S. C. REM, not incubation, improves creativity by priming associative networks. Proc. Natl. Acad. Sci. 106, 10130-10134 (2009).

43. Lewis, P. A., Knoblich, G. \& Poe, G. How Memory Replay in Sleep Boosts Creative Problem-Solving. Trends Cogn. Sci. 22, 491-503 (2018).

44. Landmann, N. et al. The reorganisation of memory during sleep. Sleep Med. Rev. 18, 531-541 (2014).

45. Peirce, J. et al. PsychoPy2: Experiments in behavior made easy. Behav. Res. Methods 51, 195-203 (2019).

46. Laukkonen, R. E. \& Tangen, J. M. How to detect insight moments in problem solving experiments. Front. Psychol. 9, 282 (2018).

47. van Casteren, M. \& Davis, M. H. Mix, a program for pseudorandomization. Behav. Res. Methods 38, 584-589 (2006). 
772 48. New, B., Pallier, C., Brysbaert, M. \& Ferrand, L. Lexique 2: A new French lexical 773 database. Behav. Res. Methods Instrum. Comput. 36, 516-524 (2004).

774 49. Boccaletti, S., Latora, V., Moreno, Y., Chavez, M. \& Hwang, D. Complex networks: 775 Structure and dynamics. Phys. Rep. 424, 175-308 (2006).

776 50. Oldham, S. \& Fornito, A. The development of brain network hubs. Dev. Cogn. 777 Neurosci. 36, 100607 (2019).

778 51. Christensen, A. P., Kenett, Y. N., Aste, T., Silvia, P. J. \& Kwapil, T. R. Network 779 structure of the Wisconsin Schizotypy Scales-Short Forms: Examining psychometric network 780 filtering approaches. Behav. Res. Methods 50, 2531-2550 (2018). 


\section{Supplementary material}

\section{SI1: Riddle-specific RJT material}

Here, we specified how we built the verbal material for the RJT task that was specific to each riddle. It consisted of a list of 20 different words (four different lists for Zoe, Daniel, Car, and Bar riddles). The same list was used in the RJT performed before and after working on a given riddle. To create those lists, we proceeded in two successive steps.

First, three creativity experts (TB, DO, EV) independently proposed up to 30 words for each riddle (blind procedure). To do so, we followed the approach used by Durso et al. ${ }^{12}$, consisting in selecting words that were explicitly stated in the problem (e.g., bar and shotgun in the Bar riddle), related to the solution (e.g., remedy and relieve in the Bar riddle), or usually associated with the problem but not with the solution (e.g., drunk and loaded in the Bar riddle). Words could be verbs, nouns, or adjectives. To ensure that selected words could be easily accessible in individuals' SemNets, we only chose frequent words, with a lexical frequency higher than one million occurrences using the lexicon database (http://www.lexique.org/; ${ }^{44}$ ).

Second, the three experts shared their respective lists and reached a consensus for 20 words per riddle (including $4 \pm 0.8$ words explicit to the problem, $6 \pm 1$ words related to the solution, and $10 \pm 1$ words loosely associated with the problem but unrelated to the solution). During this selection process, we were careful to avoid words that were too closely related to the solution (e.g., game in the Zoe riddle), too strongly associated with other ones (e.g., bar and barman in the Bar riddle), or too distant from all of the other words within the same list. In this step, we chose 20 words (rather than 14 in the Durso et al. study ${ }^{12}$ ) to obtain a bigger SemNet (larger graphs are ideal for finding subtle differences in global metrics). As a side note, for the Bar riddle, we selected 10 out of the 14 words that were already used in the Durso et al. study ${ }^{12}$ with ten novel words. The decision to exclude four words (paper bag, pretzel, man, and barman) from the original list was based on pilot experiments (e.g., no participants thought of paper bag as a remedy for hiccup; pretzel was too isolated in the network, and man and barman were too strongly associated with each other and with the word bar).

\section{SI2: Creativity tasks}

During the 4h-experiment, participants completed several creativity tasks. All tasks were computed using the Psychopy software ${ }^{46}$ running on individual computers (except for the ATTA that used paper and pencil material) in a classroom dedicated to cognitive experiments. Task-related instructions were explained before the start of each task.

\section{The Combined Associates Task (CAT; $\left.{ }^{34,35}\right)$}

The CAT consists in finding a word connecting three given unrelated cue words. Semantic association strength between the expected solution and the three cue words was controlled for each trial. Hence, distant trials (i.e., trials with a distant solution) and close trials (i.e., trials with a close solution) were built. According to the associative theory of creativity, the more remote the elements to be combined (i.e., distant trials), the more creative the process.

Participants underwent 40 trials (20 distant trials and 20 close trials) in random order. They had up to 30 seconds to write their answer with the keyboard. To explore individual 
creative abilities, we considered the number of correct responses in distant trials (CAT-dist) and computed a $C A T$-index (corresponding to the difference between performance on close and distant trials, divided by the mean performance in both conditions). The CAT-index reflects the ability to solve distant trials (the more creative condition) when controlling for performance in the less creative condition (close trials): the lower the CAT-index, the higher the creativity abilities.

\section{The Abbreviated Torrance Test for Adult $\left(\right.$ ATTA; $\left.{ }^{36}\right)$}

The ATTA includes three activities (one verbal and two figural). In the first activity, participants had to generate as many ideas as they could related to an open-ended problem ("Suppose that you can walk on air or fly. Imagine what problem you might encounter"). In the second activity, participants were asked to draw pictures as unusual as possible by using two incomplete figures. In the last activity participants had to see how many pictures or objects they could draw using triangles.

Participants were asked to perform each activity one at a time with a pencil on a dedicated piece of paper. They had 3 minutes per activity. Before starting, instruction was read orally to participants. Participants were evaluated on their productions' fluency, originality, elaboration, flexibility, and other indicators according to the ATTA manual. A composite index (ATTA-index) was computed based on all scaled scores: the higher the ATTA-index, the higher the creativity abilities.

\section{The Inventory of Creative Activities and Achievements $\left(I C A A ;{ }^{37}\right)$}

The ICAA is a self-questionnaire assessing creative activities and achievements across eight real-life creative domains (e.g., literature, music, art and craft, cooking, sport, visual art, performing arts, science, and engineering). The creative activities $(C-A c t)$ score reflects the frequency in which participants engaged in various creative activities. Six different questions were asked for each domain, and participants reported the frequency with which they engaged in each activity during the last ten years, using a scale ranging from 0 (never) to 4 (more than ten times). For each participant, the final domain-general score of $C$-Act was the sum of the creative activities across all activities of the eight different domains. The creative achievements $(C$-Ach $)$ score estimated the level of achievement reached in all creative domains. Ten different levels of achievement were included for each domain going from 0 (never engaged in this domain) to 10 (I have already sold some of my work in this domain). For each participant, the final domain-general score of $C$-Ach was the sum of the scores across the eight different domains. 


\section{SI3: Creative problem-solving assessed by our riddles relates to creative abilities}

876

877

878

In order to assess how much our riddles represent creative problems, we explored the relationship between the ability to solve the four riddles (i.e., the individual solving rate across the four riddles) and behavioral variables of creativity tasks. Relations were assessed with several Spearman correlations, corrected for multiple comparisons with FDR correction. Correlations that survive the FDR correction need to maintain their $p_{\text {corr }}$ under .05.

We found that riddle-solving rate was positively and significantly correlated with the CAT-dist $\left(\mathrm{rho}=.33, p_{\text {corr }}=2.8110^{-3}\right)$, the ATTA-index $\left(\mathrm{rho}=.29, p_{\text {corr }}=4.7110^{-3}\right)$, the $C$-Ach $\left(\mathrm{rho}=.29, p_{\text {corr }}=4.7110^{-3}\right)$, and the $C-A c t\left(\mathrm{rho}=0.27, p_{\text {corr }}=7.7510^{-3}\right)$. It was negatively and significantly correlated with the CAT-index (rho $=-.33, p_{\text {corr }}=2.8110^{-3}$; Figure S2). These results strongly suggest that our riddles' material measures creative thinking and refers to creative problem-solving. 

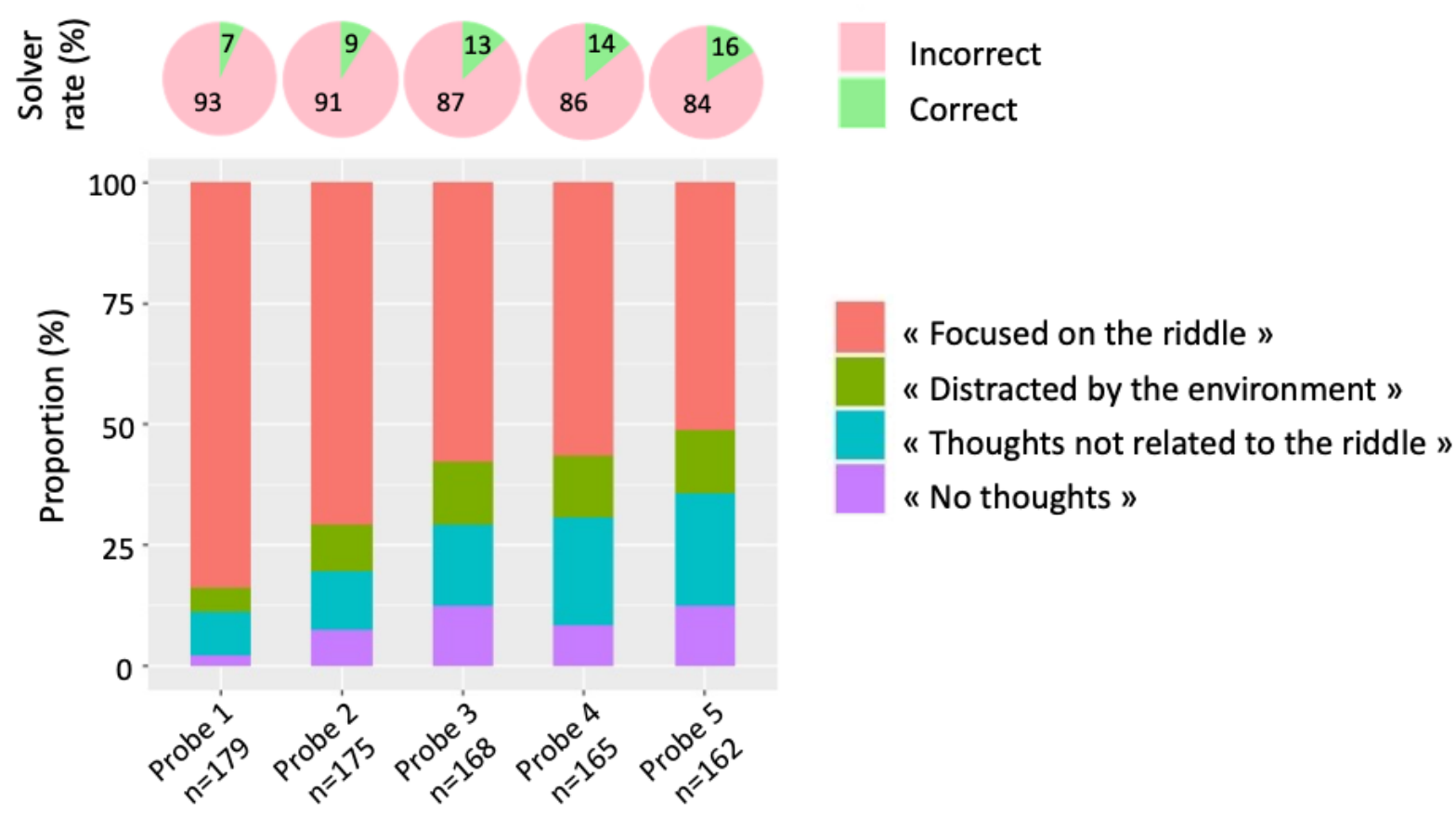

SI Figure S1. Attentional focus during the thinking phase over time. Proportion of responses to each attentional probe (one every 2 minutes, 5 in total) for each category ("Focused on the riddle" in red, "Distracted by the environment" in green, "Thoughts not related to the riddle" in blue and "No thoughts" in purple) is indicated by the bar plots. This proportion was calculated by adding the total number of non-solvers' responses for each category across all four riddles, divided by the total number of non-solvers (n) at one given probe. Proportion of solvers (in green) and non-solvers (in red) over time is indicated by the pie charts above the corresponding bar plot (all riddles combined). 


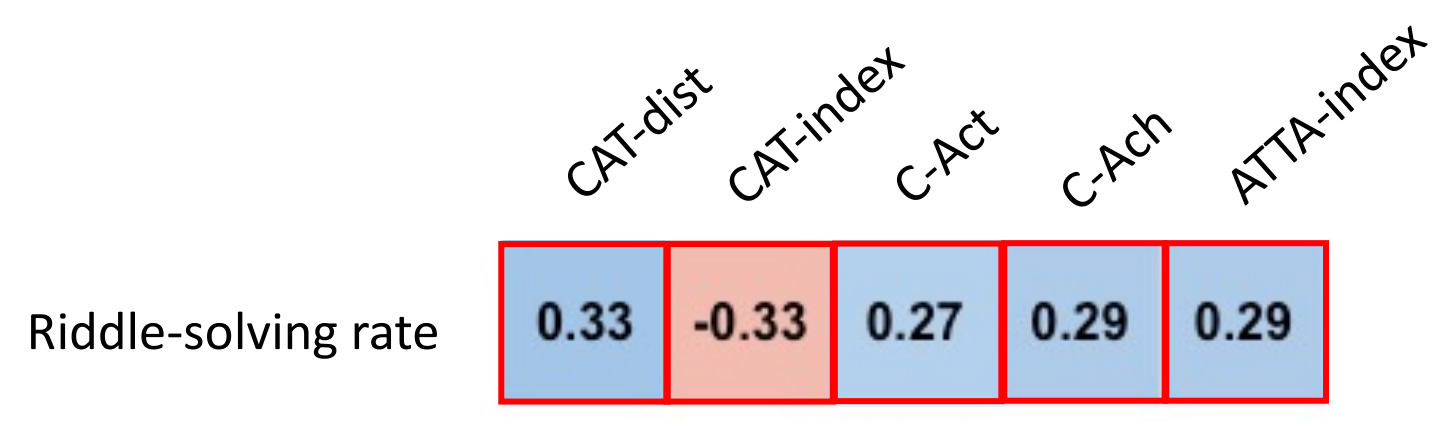

rho

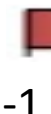

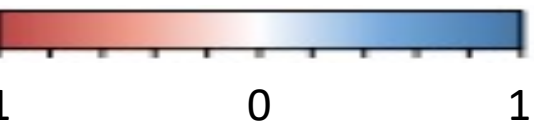

$$
\mathrm{p}_{\text {corr }}<.05
$$

(FDR correction

SI Figure S2. Correlation matrix between riddle-solving rate and creativity tasks. Correlation coefficients are indicated in the matrix (rho value, Spearman correlation) and with the color bar. Significant correlations after FDR correction $\left(p_{\text {corr }}<.05\right)$ are highlighted with a red square. CAT-dist refer to the number of correct distant trials in the CAT and CAT-index to the difference in performance between close and distant trials (the lower the CAT-index, the higher creativity abilities). $C$-Act and $C$-Ach refer to the involvement in creative activities and achievements in the ICAA, respectively. ATTA-index refers to the final combined and scaled 909 score of ATTA. 


\section{Solver group}
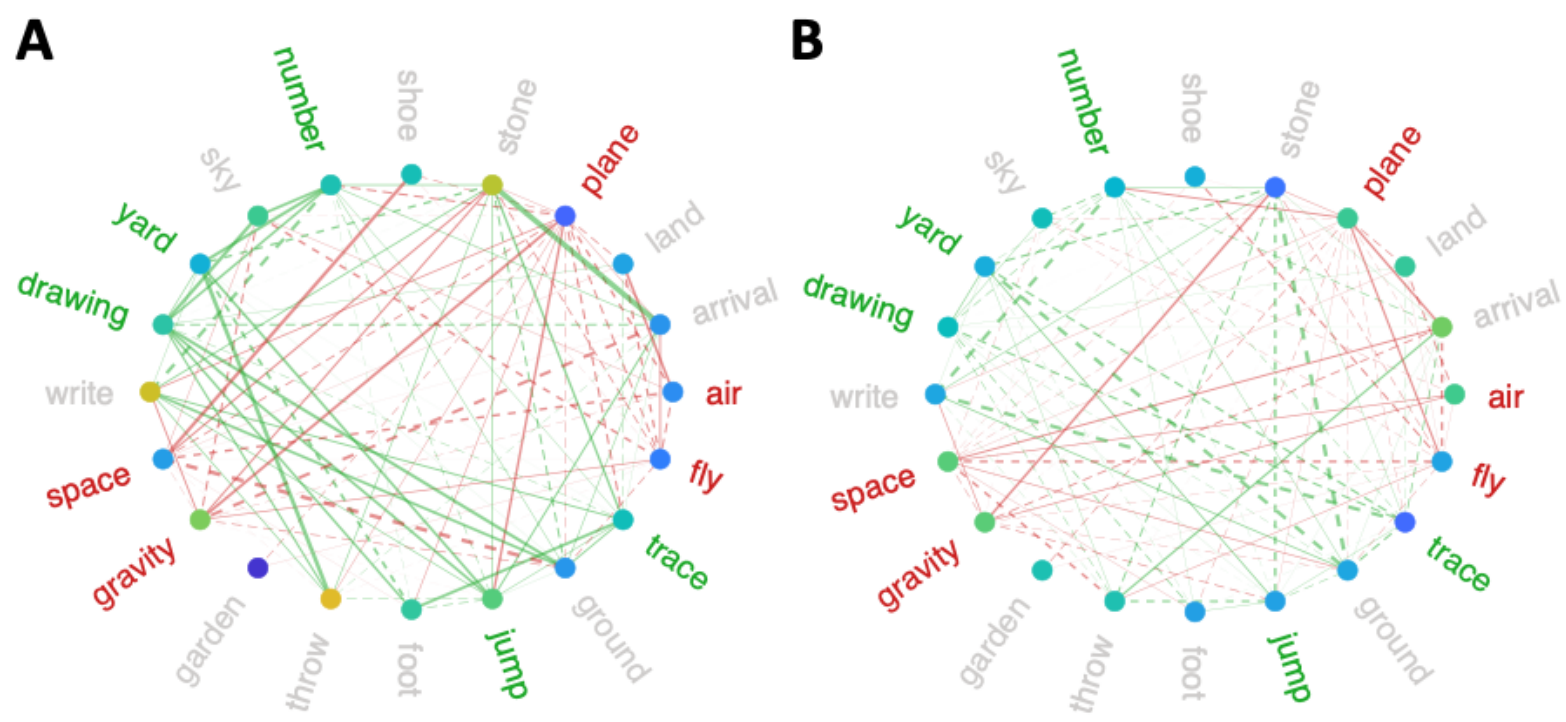

Misleading word pairs/words - - - Negative $\Delta \mathrm{W}_{\text {local }}$
Helpful word pairs/words
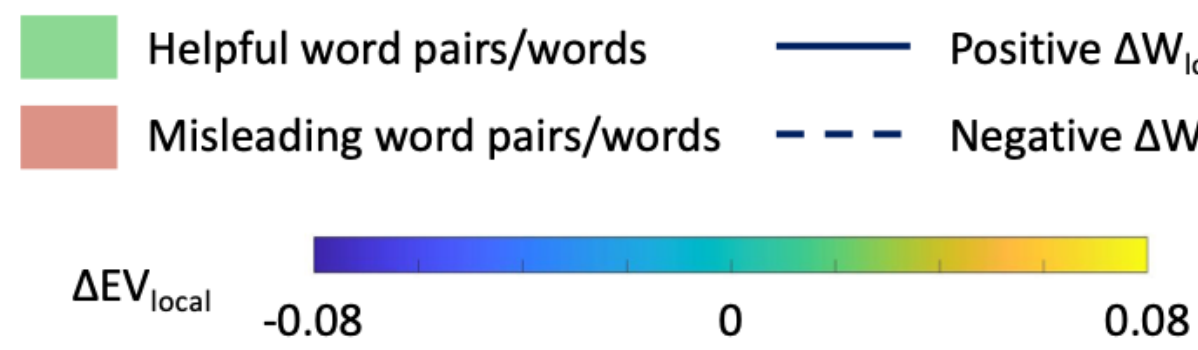

SI Figure S3. Changes in SemNets at the group level. $\Delta \mathrm{W}_{\text {local }}$ and $\Delta E V_{\text {local }}$ for Zoe riddle were averaged across individuals in the solver group (A) and the non-solver group (B). SemNets are displayed using a circle layout in which each node has the same position. Colors of the node labels represent their categorization as helpful (in green) or misleading (in red) in solving the problem. We categorized the pairs and words as helpful or misleading based on the distribution of the impact ratings (Figure S4 and S5): scores of pairs/words in the first quartile were labeled as misleading ( $n=48$ pairs and $n=5$ nodes respectively) whereas those in the last quartile were labeled as helpful ( $\mathrm{n}=48$ pairs and $\mathrm{n}=5$ nodes respectively). Node colors represent $\Delta \mathrm{EV}$ local from negative values (i.e., $\mathrm{EV}_{\text {PreSemNet }}>\mathrm{EV}_{\text {PostSemNet; }}$ in blue) to positive values (i.e., $\mathrm{EV}_{\text {PostSemNet }}$ $>\mathrm{EV}_{\mathrm{PreSemNet}}$; in yellow). Only helpful and misleading edges are shown (respectively in green and red), and their thickness is proportional to $\Delta \mathrm{W}_{\text {local }}$ (the wider the line, the higher $\Delta \mathrm{W}_{\text {local }}$ ). Dashed lines represent negative $\Delta \mathrm{W}_{\text {local }}$ (i.e., $\mathrm{W}_{\text {PreSemNet }}>\mathrm{W}_{\text {PostSemNet }}$ ), whereas solid ones represent positive $\Delta \mathrm{W}_{\text {local }}$ (i.e., $\mathrm{W}_{\text {PostSemNet }}>\mathrm{W}_{\text {PreSemNet }}$ ). We can observe that more changes occurred in the solvers' SemNet than the non-solvers one, suggesting a higher restructuration related to solving. Positive changes were mainly observed in helpful nodes and edges in the solvers' network. 

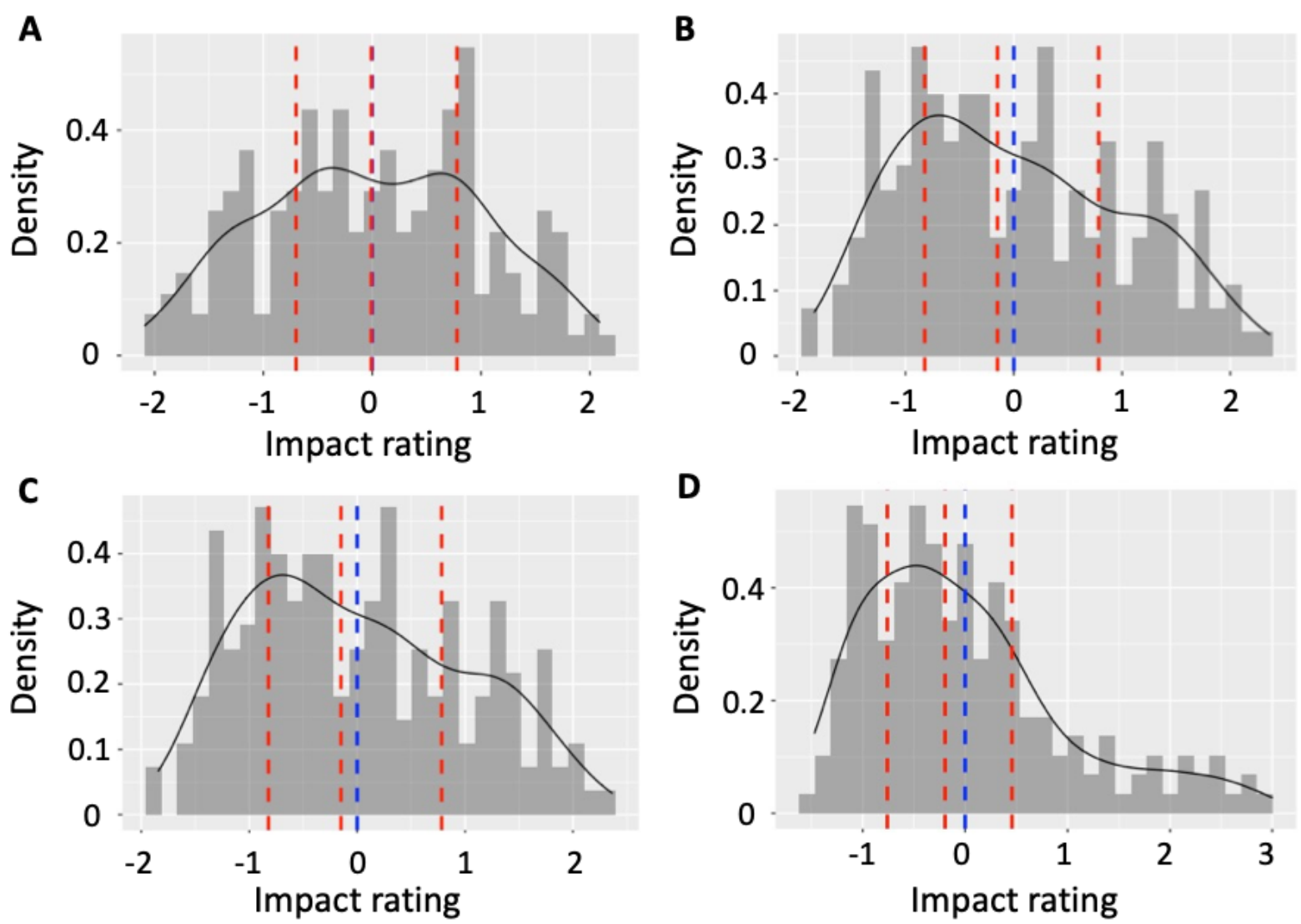

SI Figure S4. Impact ratings of riddles-related word pairs. Distribution of the averaged scores for each word pair ( $\mathrm{n}=190)$ across the nine judges for Zoe (A), Car (B), Bar (C) and Daniel riddles (D). Scores were normalized (z-scored) within each riddle. Blue dashed line indicates the mean and red ones the median and quartiles. 

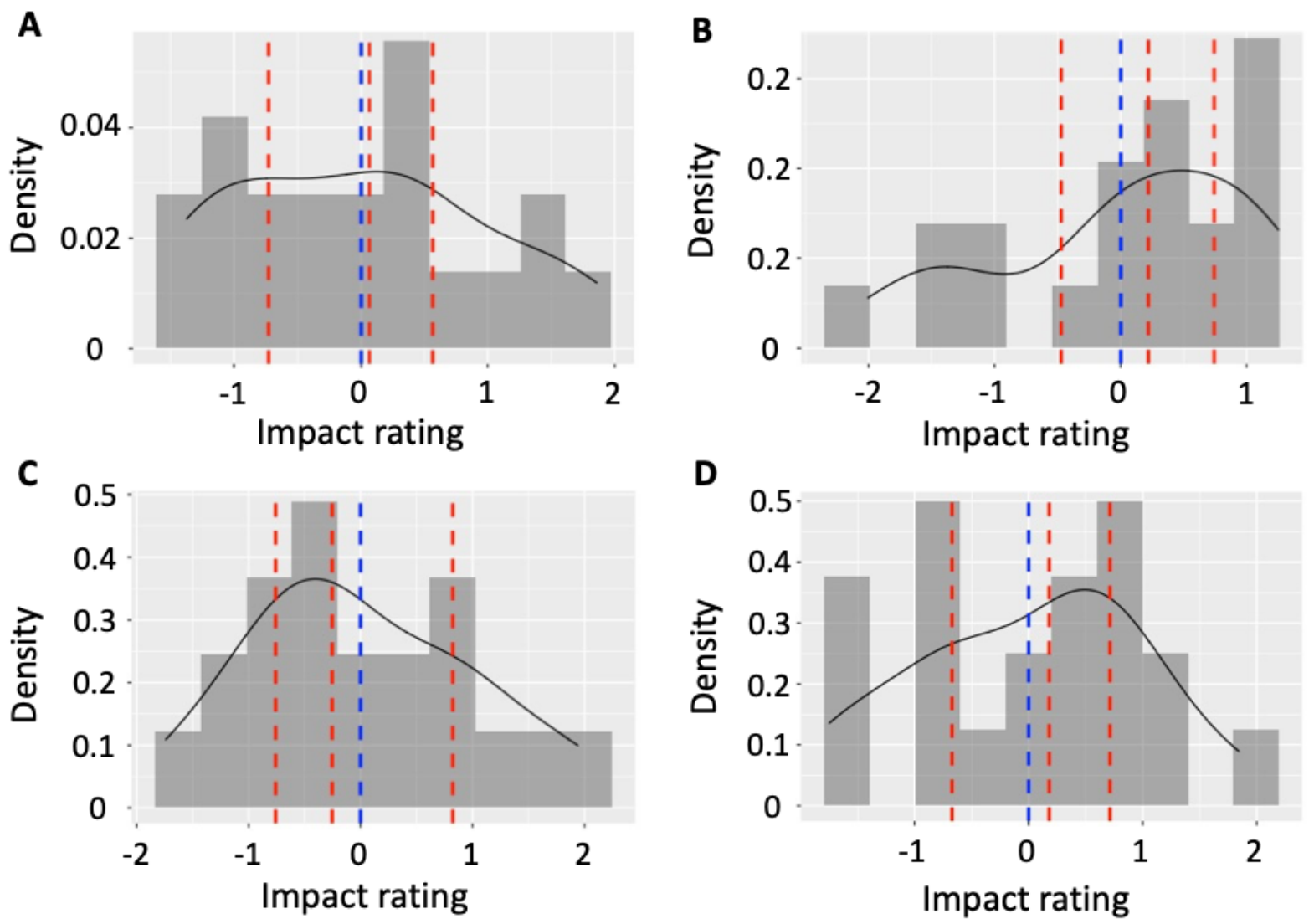

SI Figure S5. Impact ratings for riddles-related words. Distribution of indirect impact ratings calculated for each word $(\mathrm{n}=20)$ and riddle (Zoe, A; Car, B; Bar, C; and Daniel, D). We averaged the impact rating of the pairs involving this word $(n=19$ pairs per words) for each word. Scores were normalized (z-scored) within each riddle. Blue dashed line represents the mean and red ones the quartiles. 


\section{Problem statement}

\section{Zoe riddle}

French Zoé jette une pierre qui atterrit dans le ciel. Comment est-ce possible et dans quel contexte?

English Zoe throws a stone that lands in the sky. How is it possible and in which context?

\section{Car riddle}

French Un homme pousse sa voiture. Il s'arrête devant un hôtel et se sait alors totalement ruiné. Pourquoi?

English A man pushes his car in front of a hotel. Instantly he knows he's bankrupt. What's going on?

\section{Bar riddle}

French Un homme entre dans un bar et se dirige vers le comptoir pour demander un verre d'eau. Le barman sort un pistolet et le pointe vers l'homme. Ce dernier dit « Merci ! » et s'en va. Comment expliquer la situation?

English A man walks into a bar and ask for a glass of water. The bartender points a shotgun at the man. The man says "Thank you" and walks out. How to make sense of this story?

\section{Daniel riddle}

French Daniel croise dans la rue un couple qui marche vers lui. Alors qu'ils ne se connaissent pas, Daniel tape violement l'homme. Sa femme à ses côtés est paniquée. L'homme est sous le choc, mais Daniel est fier de lui. Pourquoi ?

English Daniel meets a couple walking towards him in the street. Although they don't know each other, Daniel violently hits the man. His wife at his side is panicked. The man is in shock, but Daniel is proud of himself. Why?

SI Table S1. Riddle. Riddles used in our experiment with its related English translation. Solutions are provided in a separate table (Table S2). 


\section{Problem response}

\begin{tabular}{|c|c|}
\hline \multicolumn{2}{|c|}{ Zoe riddle } \\
\hline French & Zoé joue à la marelle \\
\hline English & Zoe plays hopscotch \\
\hline \multicolumn{2}{|c|}{ Car riddle } \\
\hline French & L'homme joue au Monopoly \\
\hline English & The man plays Monopoly \\
\hline \multicolumn{2}{|c|}{ Bar riddle } \\
\hline French & L'homme a le hoquet \\
\hline English & The man has hiccups \\
\hline \multicolumn{2}{|c|}{ Daniel riddle } \\
\hline French & L'homme était en train de s'étouffer \\
\hline English & The man was suffocating \\
\hline
\end{tabular}

949

950

951 SI Table S2. Riddle answers. Hopscotch is a famous backyard children game in which you 952 have to throw a stone in square drawn on the ground, and then hop on them. In France, the last 953 square is traditionally represented and named as the sky (Figure 1). 


\begin{tabular}{llllllll} 
Zoe riddle & & Car riddle & Bar riddle & \multicolumn{3}{c}{ Daniel riddle } \\
\hline French & English & French & English & French & English & French & English \\
\hline air & air & acheter & to buy & bar & bar & agression & attack \\
arrivée & arrival & arrêter & to stop/arrest & boire & to drink & amour & love \\
atterrir & to land & banque & bank & bruit & noise & bleu & blue \\
avion & plane & billet & bill & chargé & loaded & choc & shock \\
caillou & stone & carte & card & comptoir & counter & cour & heart \\
chaussure & shoe & casino & casino & danger & danger & coup & hit \\
chiffre & number & chance & luck & détente & rest/trigger & couple & couple \\
ciel & sky & départ & departure & dispute & quarrel & crier & to yell \\
cour & yard & dormir & sleep & ivre & drunk & dispute & quarrel \\
dessin & drawing & emprunt & loan & menace & threat & dos & back \\
écrire & to write & fortune & fortune & merci & thanks & douleur & pain \\
espace & space & gare & station & mourir & to die & fou & mad \\
gravité & gravity & hôtel & hotel & peur & fear & gorge & throat \\
jardin & garden & paix & peace & pistolet & shotgun & jaloux & jealous \\
lancer & to throw & police & police & remède & remedy & rouge & red \\
pied & foot & prison & jail & soulagé & relieved & sang & blood \\
sauter & to jump & roue & wheel & surprise & surprise & secours & rescue \\
sol & ground & ruiné & ruined & sympa & friendly & soulager & to relieve \\
tracer & to trace & vacances & vacation & télé & television & tromper & to cheat \\
voler & to fly & voiture & car & verre d'eau & glass of water & violent & violent \\
\hline & & & & & & &
\end{tabular}

956 SI Table S3. Riddle-specific word lists used in the RJT. Lists of 20 words preselected for 957 each riddle with their respective English translations. 


\section{$\beta \quad$ SE $\quad$ v value p value}

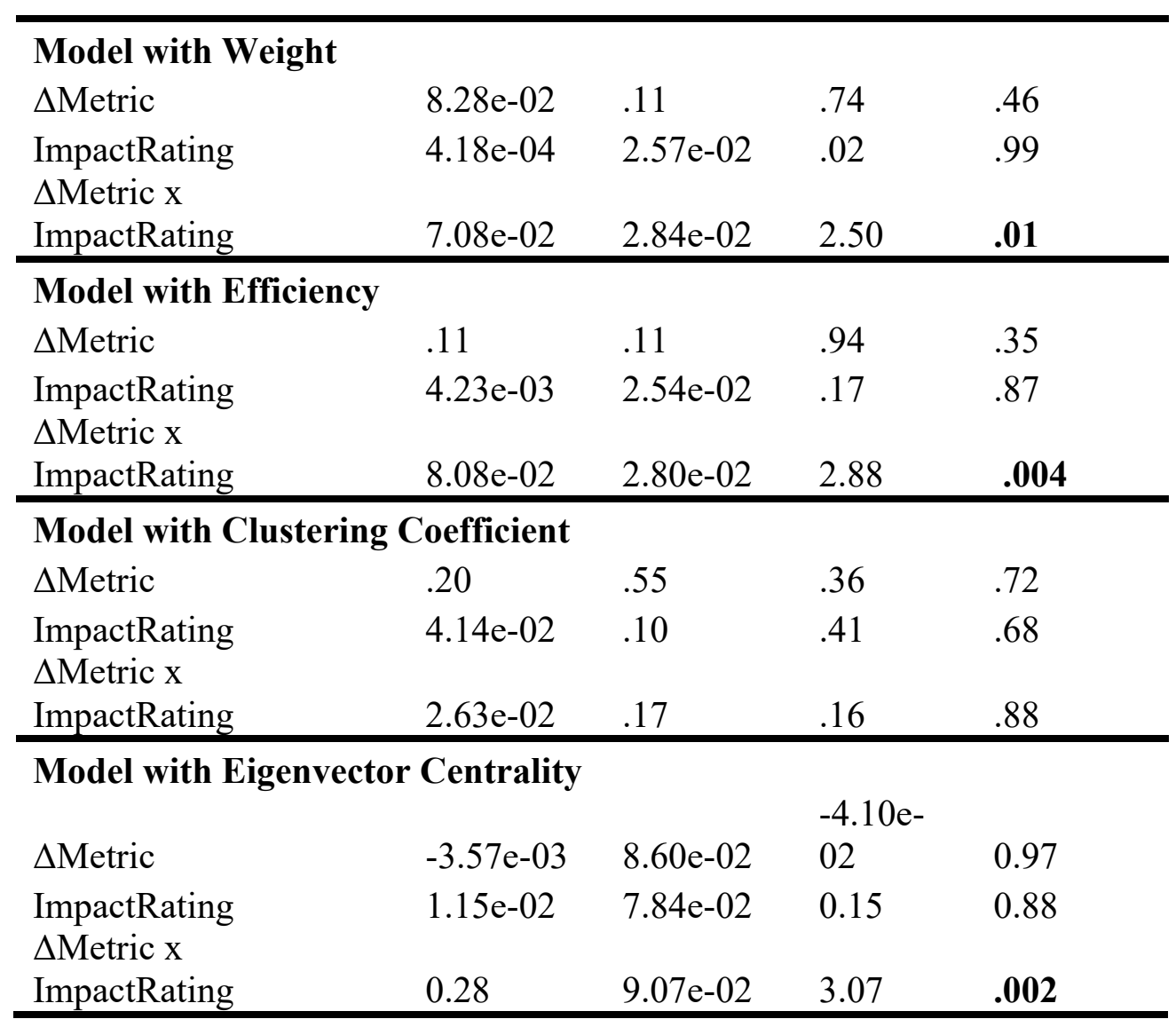

SI Table S4. Results of nonlinear mixed model analyses. $\Delta$ Metric represents the difference between the considered metric between the PreSemNet and the PostSemNet. ImpactRating corresponds either to the importance of edges or nodes for solving the problem based on independent assessment. $\Delta$ Metric $\mathrm{x}$ ImpactRating is the interaction between these parameters. We ran a mixed model for each metric. Significant results $(p<.05)$ are in bold. 